\title{
Spencer Cohomology and 11-Dimensional Supergravity
}

\author{
José Figueroa-O'Farrill, Andrea Santi \\ Maxwell Institute and School of Mathematics, The University of Edinburgh, James Clerk Maxwell Building, \\ Peter Guthrie Tait Road, Edinburgh, EH9 3FD, UK. E-mail: J.M.Figueroa@ed.ac.uk; asanti.math@gmail.com
}

Received: 27 November 2015 / Accepted: 12 May 2016

Published online: 29 June 2016- (C) The Author(s) 2016. This article is published with open access at Springerlink.com

\begin{abstract}
We recover the classification of the maximally supersymmetric bosonic backgrounds of 11-dimensional supergravity by Lie algebraic means. We classify all filtered deformations of the $\mathbb{Z}$-graded subalgebras $\mathfrak{h}=\mathfrak{h}_{-2} \oplus \mathfrak{h}_{-1} \oplus \mathfrak{h}_{0}$ of the Poincaré superalgebra $\mathfrak{g}=\mathfrak{g}_{-2} \oplus \mathfrak{g}_{-1} \oplus \mathfrak{g}_{0}=\mathrm{V} \oplus \mathrm{S} \oplus \mathfrak{s o}(\mathrm{V})$ which differ only in zero degree, that is $\mathfrak{h}_{0} \subset \mathfrak{g}_{0}$ and $\mathfrak{h}_{\mathfrak{j}}=\mathfrak{g}_{j}$ for $\mathfrak{j}<0$. Aside from the Poincaré superalgebra itself and its $\mathbb{Z}$-graded subalgebras, there are only three other Lie superalgebras, which are the symmetry superalgebras of the non-flat maximally supersymmetric backgrounds. In passing we identify the gravitino variation with (a component of) a Spencer cocycle.
\end{abstract}

\section{Contents}

1. Introduction . . . . . . . . . . . . . . . . . . 628

2. The Deformation Complex . . . . . . . . . . . . . . . . 632

2.1 The Poincaré superalgebra . . . . . . . . . . . . . . . . . . . . . . . . . . . . . . 632

2.2 The Spencer complex . . . . . . . . . . . . . . . . . 633

3. Infinitesimal Deformations . . . . . . . . . . . . . . 635

3.1 Infinitesimal deformations of $\mathfrak{g} \ldots \ldots \ldots 636$

3.2 Infinitesimal deformations of subalgebras $\mathfrak{h} \subset \mathfrak{g} \ldots \ldots . \ldots 642$

4. Integrating the Deformations . . . . . . . . . . . . . . . . . . . . . . . . . . . . . . 644

4.1 The non-trivial deformations . . . . . . . . . . . . . . . . . 644

4.2 First-order integrability of the deformation . . . . . . . . . . . . . . . . 649

4.3 All-orders integrability of the deformation . . . . . . . . . . . . . 650

5. Discussion and Conclusion . . . . . . . . . . . . . . . . 654

Appendix A: Clifford Conventions . . . . . . . . . . . . . . . . . 656

Appendix B: Some Representations of $\mathfrak{s o}(\mathrm{V}) \ldots \ldots \ldots$. . . . . . . . . . 658

References ......................... 659

EMPG-15-22. 


\section{Introduction}

The work described in this paper is an attempt at breaking new ground in the classification problem of supersymmetric backgrounds of 11-dimensional supergravity. This problem as such has been pursued on and off for the last 15 years; although its roots date back to the 1980s and the classification results for Freund-Rubin-like backgrounds (see, e.g., the review [1]) in the context of Kaluza-Klein supergravity.

A convenient organising principle in the classification of supersymmetric supergravity backgrounds is the fraction $v$ of supersymmetry preserved by the background, which is "categorified" as the dimension $\mathrm{N}=32 v$ of the odd subspace of its Killing superalgebra. At present there exist a classification for $N=32$ [2], non-existence results for $N=31[3,4]$ and $N=30[5]$, a structure result for $N=1[6,7]$, and a huge zoo of solutions for other values of $\mathrm{N}$, but no claim of classification. No solutions are known for $30>N>26$, but there is a pp-wave background with $N=26$ [8]. This "supersymmetry gap" is reminiscent of the gap phenomenon in geometric structures (see, e.g., $[9,10]$ ) and, indeed, part of the motivation to explore the approach presented in this paper was to understand the nature of this gap.

The consensus seems to be that, at present, the classification of all supersymmetric backgrounds is inaccessible, whereas that of highly supersymmetric backgrounds seems tantalisingly in reach. In particular, backgrounds with $\mathrm{N}>16$ are now known to be locally homogeneous [11] and this brings to bear the techniques of homogeneous geometry to classify certain kinds of backgrounds; e.g., symmetric $[12,13]$ or homogeneous under a given Lie group [14,15], at least when the group is semisimple.

This paper is a first step in a Lie algebraic approach at the classification problem. The proposal, to be made more precise in a forthcoming paper, is to take the Killing superalgebra as the organising principle. As we will show in that forthcoming paper, the Killing superalgebra of a supersymmetric 11-dimensional supergravity background (and also, indeed, of other supergravity theories) is a filtered deformation of a subalgebra of the relevant Poincare superalgebra. The classification problem of filtered deformations of Lie superalgebras seems tractable via cohomological techniques [16,17] which extend the use of Spencer cohomology in the theory of G-structures or, more generally, Tanaka structures.

Therefore in this paper we will present a Lie algebraic derivation of (the symmetry superalgebras of) the maximally supersymmetric bosonic backgrounds of 11-dimensional supergravity by purely representation theoretic means. In so doing we will actually "rediscover" 11-dimensional supergravity from a cohomological calculation.

Our point of departure will be the Poincaré superalgebra $\mathfrak{g}=\mathfrak{g}_{-2} \oplus \mathfrak{g}_{-1} \oplus \mathfrak{g}_{0}=$ $\mathrm{V} \oplus \mathrm{S} \oplus \mathfrak{s o}(\mathrm{V})$ or, more precisely, its supertranslation ideal $\mathfrak{m}=\mathfrak{m}_{-2} \oplus \mathfrak{m}_{-1}=\mathrm{V} \oplus \mathrm{S}$. At first, it might seem overoptimistic to expect that such a derivation is possible. How does the supertranslation ideal (or even the Poincaré superalgebra) know about the maximally supersymmetric supergravity backgrounds? We can give at least two heuristic answers to this question.

The physicist's answer is that, in a sense, this has always been possible, albeit via a rather circuitous route. That route starts by searching for massless irreducible unitary representations of the Poincaré superalgebra. Following Nahm [18], we would find the "supergravity multiplet": the unitary irreducible representation induced from the (reducible) representation of the "little group" $\operatorname{Spin}(9)$ isomorphic to

$$
\odot_{0}^{2} \mathrm{~W} \oplus \Lambda^{3} \mathrm{~W} \oplus(\mathrm{W} \otimes \Sigma)_{0},
$$


where $W$ and $\Sigma$ are, respectively, the real 9-dimensional vector and 16-dimensional spinor representation of $\operatorname{Spin}(9), \odot_{0}^{2}$ means symmetric traceless and the subscript 0 on the last term means the kernel of the Clifford action $W \otimes \Sigma \rightarrow \Sigma$ or, equivalently, "gamma traceless". (More generally, we use the notation $\odot^{n}$ to mean the $n$-th symmetric tensor power.) In this data, a physicist would recognise at once the physical degrees of freedom corresponding to a Lorentzian metric g, a 3 -form potential $A$ and a gravitino $\Psi$ and would set to construct a supergravity theory with that field content. It turns out that there is a unique such supergravity theory, which was constructed by Cremmer, Julia and Scherk in [19]. The action (with $\Psi=0$ ) is given by the sum

$$
\mathrm{I}=\mathrm{I}_{\mathrm{EH}}+\mathrm{I}_{\mathrm{M}}+\mathrm{I}_{\mathrm{CS}}=\frac{1}{2} \int \mathrm{Rdvol}+\frac{1}{4} \int \mathrm{F} \wedge \star \mathrm{F}+\frac{1}{12} \int \mathrm{F} \wedge \mathrm{F} \wedge A
$$

where $F=d A$, of an Einstein-Hilbert, Maxwell and Chern-Simons actions. The full action (including the terms depending on the gravitino $\Psi$ ) is invariant under local supersymmetry. The transformation of the gravitino under local supersymmetry defines a connection $\mathrm{D}$ on the spinor bundle, which encodes most of the geometric data of the supergravity theory. For all vector fields $X$ and spinor fields $\varepsilon$, the connection $D$ is defined by

$$
D_{X} \varepsilon=\nabla_{X} \varepsilon+\frac{1}{6} \iota_{X} F \cdot \varepsilon+\frac{1}{12} X^{b} \wedge F \cdot \varepsilon
$$

with $X^{b}$ the dual one-form to $X$ and · denoting the Clifford action.

A maximally supersymmetric bosonic background is one where $\Psi=0$ and $\mathrm{D}$ is flat (one checks that D-flatness actually implies the field equations). The D-flatness equations can be solved and one finds, as was done in [2], that besides Minkowski spacetime (with $\mathrm{F}=0$ ) there are three further families of backgrounds: two one-parameter families of Freund-Rubin backgrounds - the original background $\mathrm{AdS}_{4} \times \mathrm{S}^{7}$ found by Freund and Rubin in [20] and $\mathrm{AdS}_{7} \times \mathrm{S}^{4}$, found by Pilch et al. in [21] - and a symmetric ppwave found by Kowalski-Glikman in [22] and interpreted in [23] as the Penrose limit of the Freund-Rubin backgrounds. The calculation of the symmetry superalgebra of these backgrounds is then straightforward and we arrive at the Poincaré superalgebra itself for the Minkowski background, orthosymplectic Lie superalgebras osp (8|4) for $\operatorname{AdS}_{4} \times \mathrm{S}^{7}$ and $\mathfrak{o s p}(2,6 \mid 4)$ for $\mathrm{AdS}_{7} \times \mathrm{S}^{4}$, and a contraction thereof for the Kowalski-Glikman wave (see [24-26]). Although all of these backgrounds are maximally supersymmetric, it is the Minkowski background which has the largest symmetry: the Poincaré superalgebra has dimension (66|32), whereas the symmetry superalgebras of the other maximally supersymmetric bosonic backgrounds have dimension (38|32).

That would be the physicist's answer, but there is also a geometer's answer to the question of how the supertranslation ideal knows about (the symmetry superalgebras of) the maximally supersymmetric backgrounds, stemming from the integrability problem for geometric structures. The point of departure in this story is the fact that 11-dimensional supergravity also admits, besides the traditional "component" formulation, a geometric presentation in terms of supermanifolds. This usually amounts to giving a reduction to $\operatorname{Spin}(V)$ of the linear frame bundle of a supermanifold $M$ of dimension (11|32) or, in other words, a G-structure $\pi: P \rightarrow M$ where the structure group $G=\operatorname{Spin}(V)$ acts on the vector space direct sum $V \oplus S$ of its vector and spinor representations (see $[27,28]$ ). The geometric structure under consideration is not arbitrary but it satisfies some constraints, expressed in terms of appropriate nondegeneracy conditions on the intrinsic torsion of $\pi: P \rightarrow M$ (see, e.g., [29] for a geometric motivation of the constraints). The constraints 
put the theory "on-shell", in the sense that every G-structure as above gives rise to a solution of the field equations (see [30]).

It appears therefore that the "vacuum solution" given by the super Minkowski spacetime is described by a geometric structure that is not integrable or flat, at least in the sense of G-structures. In particular (the super-analogues of) the classical Spencer cohomology groups and their associated intrinsic curvatures considered in [31] are not applicable to the study of the deformations of these structures and, ultimately, to the quest for supergravity backgrounds.

In $[32,33]$ a description of 11-dimensional supergravity based on the notion of a super Poincaré structure is proposed. This is an odd distribution $\mathcal{D} \subset \mathrm{TM}$ on a supermanifold $M$ of dimension (11|32) which is of rank (0|32) and with Levi form

$$
\mathcal{L}: \mathcal{D} \otimes \mathcal{D} \rightarrow \mathrm{TM} / \mathcal{D}, \quad \mathcal{L}(\mathrm{X}, \mathrm{Y})=[\mathrm{X}, \mathrm{Y}] \bmod \mathcal{D},
$$

locally identifiable with the bracket $S \otimes S \rightarrow V$ of the supertranslation algebra $m$. Note that $\mathcal{D}$ is a maximally nonintegrable distribution and it is of depth $d=2$, in the sense that $\Gamma(\mathcal{D})+[\Gamma(\mathcal{D}), \Gamma(\mathcal{D})]=\mathfrak{X}(M)$. These structures can be studied with (the analogues for supermanifolds of) the standard techniques of the theory of Tanaka structures, a powerful generalisation of G-structures found by Tanaka in $[34,35]$ to deal with geometries supported over non-integrable distributions.

Let us briefly recall the main points of Tanaka's approach. It builds on the observation that a distribution $\mathcal{D}$ on a manifold $M$ determines a filtration

$$
\mathrm{T}_{\mathrm{x}} \mathrm{M}=\mathcal{D}_{-\mathrm{d}}(\mathrm{x}) \supset \mathcal{D}_{-\mathrm{d}+1}(\mathrm{x}) \supset \cdots \supset \mathcal{D}_{-2}(\mathrm{x}) \supset \mathcal{D}_{-1}(\mathrm{x})=\mathcal{D}_{\mathrm{x}}
$$

of each tangent space $T_{x} M, \mathcal{D}_{-i}(x)$ being the subspace of $T_{x} M$ given by the values of the vector fields in $\Gamma(\mathcal{D})_{-i}=\Gamma(\mathcal{D})_{-i+1}+\left[\Gamma(\mathcal{D})_{-1}, \Gamma(\mathcal{D})_{-i+1}\right]$ and $\Gamma(\mathcal{D})_{-1}=\Gamma(\mathcal{D})$. He then noticed that the "symbol space"

$$
\mathfrak{m}(x)=\operatorname{gr}\left(T_{x} M\right)=\mathfrak{m}_{-\mathrm{d}}(x) \oplus \mathfrak{m}_{-\mathrm{d}+1}(x) \oplus \cdots \oplus \mathfrak{m}_{-2}(x) \oplus \mathfrak{m}_{-1}(x)
$$

inherits the structure of a $\mathbb{Z}$-graded Lie algebra by the commutators of vector fields and assumed, as a regularity condition, that all $\mathfrak{m}(x)$ are isomorphic to a fixed $\mathbb{Z}$-graded Lie algebra $\mathfrak{m}=\mathfrak{m}_{-\mathrm{d}} \oplus \cdots \oplus \mathfrak{m}_{-1}$ which is generated by $\mathfrak{m}_{-1}$. We call such $\mathbb{Z}$-graded Lie algebras fundamental.

To any fundamental Lie algebra $\mathfrak{m}$ one can associate a unique maximal transitive prolongation in positive degrees

$$
\mathfrak{g}^{\infty}=\bigoplus_{\mathrm{p} \in \mathbb{Z}} \mathfrak{g}_{\mathfrak{p}}^{\infty}
$$

This is a (possibly infinite-dimensional) $\mathbb{Z}$-graded Lie algebra that satisfies:

1. $\mathfrak{g}_{\mathfrak{p}}^{\infty}$ is finite-dimensional for every $p \in \mathbb{Z}$;

2. $\mathfrak{g}_{\mathfrak{p}}^{\infty}=\mathfrak{m}_{\mathfrak{p}}$ for every $-\mathrm{d} \leqslant \mathrm{p} \leqslant-1$ and $\mathfrak{g}_{\mathfrak{p}}^{\infty}=0$ for every $\mathrm{p}<-\mathrm{d}$;

3. for all $p \geqslant 0$, if $x \in \mathfrak{g}_{\mathrm{p}}^{\infty}$ is an element such that $\left[x, \mathfrak{g}_{-1}^{\infty}\right]=0$, then $x=0$ (this property is called transitivity);

4. $\mathfrak{g}^{\infty}$ is maximal with these properties.

Finally, he introduced the concept of a Tanaka structure, a $G_{0}$-reduction $\pi: P \rightarrow M$ of an appropriate $\mathrm{G}_{0}^{\infty}$-principal bundle, $\operatorname{Lie}\left(\mathrm{G}_{0}^{\infty}\right)=\mathfrak{g}_{0}^{\infty}$, consisting of linear frames defined just on the subspaces $\mathcal{D}_{\chi}$ of the $T_{\chi} M$ 's (in particular the usual G-structures are the Tanaka structures of depth $d=1$ ), and also the analogs of the Spencer cohomology groups and 
their associated intrinsic curvatures for $\mathbb{Z}$-graded Lie algebras of depth $d>1$. In this context the integrable model, which realises the maximum dimension of the algebra of symmetries, is the nilpotent and simply connected Lie group with Lie algebra $\mathfrak{m}$.

In the relevant case of supermanifolds and 11-dimensional supergravity, the symbol is just the supertranslation algebra $\mathfrak{m}$, the integrable model is the super Minkowski spacetime and a Tanaka structure on a supermanifold with symbol $\mathfrak{m}$ and structure group $\mathrm{G}_{0}=\operatorname{Spin}(\mathrm{V}) \subset \mathrm{G}_{0}^{\infty}=\mathrm{CSpin}(\mathrm{V})$ is the same as a supergravity background.

This paper considers the deformations of the super Minkowski spacetime from Tanaka's perspective and recovers the classification of maximally supersymmetric bosonic backgrounds of 11-dimensional supergravity by Lie algebraic means. Our starting point is the supertranslation algebra $\mathfrak{m}=\mathfrak{m}_{-2} \oplus \mathfrak{m}_{-1}=\mathrm{V} \oplus \mathrm{S}$ and the nontrivial result [36,37] that its maximal transitive prolongation is the extension

$$
\mathfrak{g}^{\infty}=\mathfrak{g}_{-2}^{\infty} \oplus \mathfrak{g}_{-1}^{\infty} \oplus \mathfrak{g}_{0}^{\infty}=\mathrm{V} \oplus \mathrm{S} \oplus(\mathfrak{s o}(\mathrm{V}) \oplus \mathbb{R E})
$$

of the Poincaré superalgebra $\mathfrak{g}=\mathfrak{g}_{-2} \oplus \mathfrak{g}_{-1} \oplus \mathfrak{g}_{0}=\mathrm{V} \oplus \mathrm{S} \oplus \mathfrak{s o}(\mathrm{V})$ by the grading element

$$
E \in \mathfrak{g}_{0}^{\infty} \quad \text { where }\left.\operatorname{ad}(E)\right|_{\mathfrak{g}_{j}^{\infty}}=j \operatorname{Id}_{\mathfrak{g}_{j}^{\infty}}
$$

More precisely we will show that the symmetry superalgebras of the maximally supersymmetric bosonic backgrounds correspond exactly to the filtered deformations of the $\mathbb{Z}$-graded subalgebras $\mathfrak{h}=\mathfrak{h}_{-2} \oplus \mathfrak{h}_{-1} \oplus \mathfrak{h}_{0}$ of the Poincare superalgebra which differ only in zero degree, that is $\mathfrak{h}_{0} \subset \mathfrak{g}_{0}$ and $\mathfrak{h}_{j}=\mathfrak{g}_{\mathfrak{j}}$ for $\mathfrak{j}<0$. We will make evident that a gap phenomenon arises, the dimension of the symmetry superalgebra dropping when considering non-integrable geometries, and, in doing so, we also recover the connection (1) by cohomological methods. In other words, we rediscover the basic geometric object of the supergravity theory by a cohomological calculation.

We remark that similar gaps and upper bounds on the submaximal dimension for (non-super) geometric Tanaka structures were recently derived in [9] using Kostant's version [38] of Borel-Bott-Weil theory for semisimple Lie algebras, whereas in our case we require different cohomological techniques, developed for general $\mathbb{Z}$-graded Lie superalgebras by Cheng and Kac in $[16,17]$.

The paper is organised as follows. In Sect. 2.1 we introduce the problem by defining the notion of a filtered deformation of $\mathbb{Z}$-graded subalgebras $\mathfrak{h}$ of the Poincaré superalgebra $\mathfrak{g}$ differing only in degree 0 . We observe that infinitesimal filtered deformations can be interpreted in terms of Spencer cohomology. In Sect. 2.2 we introduce the Spencer differential complex $C^{\bullet, \bullet}(\mathfrak{m}, \mathfrak{g})$ and prove that $\mathrm{H}^{\mathfrak{p}, 2}(\mathfrak{m}, \mathfrak{g})=0$ for all even $p \geqslant 4$. The main result of Sect. 3.1 is Proposition 7, giving an explicit isomorphism of $\mathfrak{s o}(\mathrm{V})$-modules between the group $\mathrm{H}^{2,2}(\mathfrak{m}, \mathfrak{g})$ and $\Lambda^{4} \mathrm{~V}$. With only a modicum of hyperbole, we explain that we may interpret this result as a cohomological derivation of 11-dimensional supergravity. In Sect. 3.2 we consider the subalgebras $\mathfrak{h}$, determine the corresponding Spencer groups $H^{p, 2}(\mathfrak{m}, \mathfrak{h})$ for all even $p \geqslant 2$ and prove Theorem 9 , which states that infinitesimal filtered deformations of $\mathfrak{h}$ are classified by $\mathfrak{h}_{0}$-invariant elements in $\mathrm{H}^{2,2}(\mathfrak{m}, \mathfrak{h})$. In Sect. 4 we determine the $\mathfrak{h}_{0}$-invariant elements in $\mathrm{H}^{2,2}(\mathfrak{m}, \mathfrak{h})$ and integrate the corresponding infinitesimal deformations. The classification of infinitesimal deformations is contained in Proposition 14 and their integrability is proved in Sects. 4.2 and 4.3. Our results are summarised in Theorem 16. The paper ends with some discussions in Sect. 5. Finally, Appendices A and B set our conventions and basic results on Clifford algebras, spinors and representations of $\mathfrak{s o}(\mathrm{V})$. 


\section{The Deformation Complex}

In this section we give the basic definitions and then prove the first results on the Spencer cohomology of the Poincaré superalgebra.

2.1. The Poincaré superalgebra. Let $\mathrm{V}$ denote a real 11-dimensional vector space with a Lorentzian inner product $\eta$ of signature $(1,10)$; that is, $\eta$ is "mostly minus". The corresponding Clifford algebra $C \ell(V) \cong C \ell(1,10) \cong \operatorname{End}\left(S_{+}\right) \oplus \operatorname{End}\left(S_{-}\right)$where $S_{ \pm}$are irreducible Clifford modules, real and of dimension 32. They are distinguished by the action of the volume element $\Gamma_{11} \in \mathrm{C} \ell(V)$, but are isomorphic as $\operatorname{Spin}(V)$ representations. We will work with $S=S_{-}$in what follows, that is we assume $\Gamma_{11} \cdot s=-s$ for all $s \in S$.

On $S$ there is a symplectic structure $\langle-,-\rangle$ satisfying

$$
\left\langle v \cdot s_{1}, s_{2}\right\rangle=-\left\langle s_{1}, v \cdot s_{2}\right\rangle,
$$

for all $s_{1}, s_{2} \in \mathrm{S}$ and $v \in \mathrm{V}$, where - refers to the Clifford action. In particular, $\langle-,-\rangle$ is $\operatorname{Spin}(V)$-invariant, making $S$ into a real symplectic representation of $\operatorname{Spin}(V)$. Taking adjoint with respect to the symplectic structure defines an anti-involution $\sigma$ on $\mathrm{Cl}(\mathrm{V})$ which, by (3), is characterised by $\left.\sigma\right|_{V}=-\operatorname{Id}_{V}$.

Let $\mathfrak{s o}(\mathrm{V})$ denote the Lie algebra of $\operatorname{Spin}(\mathrm{V})$. The (11-dimensional) Poincaré superalgebra is the $\mathbb{Z}$-graded Lie superalgebra

$$
\mathfrak{g}=\mathfrak{g}_{-2} \oplus \mathfrak{g}_{-1} \oplus \mathfrak{g}_{0},
$$

where $\mathfrak{g}_{0}=\mathfrak{s o}(\mathrm{V}), \mathfrak{g}_{-1}=\mathrm{S}$ and $\mathfrak{g}_{-2}=\mathrm{V}$. The $\mathbb{Z}$-grading is compatible with the parity, in the sense that $\mathfrak{g}_{\overline{0}}=\mathfrak{g}_{-2} \oplus \mathfrak{g}_{0}$ and $\mathfrak{g}_{\overline{1}}=\mathfrak{g}_{-1}$, and it allows only the following brackets:

- $[-,-]: \mathfrak{g}_{0} \times \mathfrak{g}_{\mathfrak{i}} \rightarrow \mathfrak{g}_{\mathfrak{i}}$, which consists of the adjoint action of $\mathfrak{s o}(\mathrm{V})$ on itself and its natural actions on $\mathrm{V}$ and $\mathrm{S}$;

- $[-,-]: \mathfrak{g}_{-1} \times \mathfrak{g}_{-1} \rightarrow \mathfrak{g}_{-2}$, which is the construction of the Dirac current of a spinor:

$$
\eta\left(v,\left[s_{1}, s_{2}\right]\right)=\left\langle\nu \cdot s_{1}, s_{2}\right\rangle,
$$

for all $s_{1}, s_{2} \in \mathrm{S}$ and $v \in \mathrm{V}$. Notice that from (3), it follows that $\left[\mathrm{s}_{1}, \mathrm{~s}_{2}\right]=\left[\mathrm{s}_{2}, \mathrm{~s}_{1}\right]$ and hence that it is determined by its restriction $[s, s]$ to the diagonal. It is a fact that for all $s \in S$, the vector $v=[s, s] \in V$, satisfies $\eta(v, v) \geqslant 0$, i.e. it is either null or timelike.

Note that the even Lie subalgebra $\mathfrak{g}_{\overline{0}}=\mathfrak{s o}(\mathrm{V}) \oplus \mathrm{V}$ is the Poincaré algebra. We will let $\mathfrak{m}=\mathfrak{m}_{-2} \oplus \mathfrak{m}_{-1}, \mathfrak{m}_{-2}=\mathfrak{g}_{-2}=\mathrm{V}, \mathfrak{m}_{-1}=\mathfrak{g}_{-1}=\mathrm{S}$ denote the (2-step nilpotent) supertranslation ideal. As it is generated by $\mathfrak{m}_{-1}, \mathfrak{m}$ is a fundamental $\mathbb{Z}$-graded Lie superalgebra.

We consider $\mathbb{Z}$-graded subalgebras $\mathfrak{h}=\mathfrak{h}_{-2} \oplus \mathfrak{h}_{-1} \oplus \mathfrak{h}_{0}$ of the Poincaré superalgebra which differ only in zero degree, that is, $\mathfrak{h} \subset \mathfrak{g}$ with $\mathfrak{h}_{0} \subset \mathfrak{g}_{0}$ and $\mathfrak{h}_{j}=\mathfrak{g}_{j}$ for $\mathfrak{j}<0$ and we seek filtered deformations of $\mathfrak{h}$. These are the Lie superalgebras $F$ with an associated compatible filtration $F_{\bullet}=\cdots \supset F_{-2} \supset F_{-1} \supset F_{0} \supset \cdots$ such that its associated $\mathbb{Z}$-graded Lie superalgebra agrees with $\mathfrak{h}$ (see, e.g., $[16,17]$ ). Any such filtration $F_{\bullet}$ is isomorphic as a vector space to the canonical filtration of $\mathfrak{h}$ given by $F_{\mathfrak{i}}=\mathfrak{h}$ for all $\mathfrak{i}<-2, F_{\mathfrak{i}}=0$ for all $i>0$ and

$$
\mathrm{F}_{-2}=\mathfrak{h}=\mathfrak{h}_{-2} \oplus \mathfrak{h}_{-1} \oplus \mathfrak{h}_{0}, \quad \mathrm{~F}_{-1}=\mathfrak{h}_{-1} \oplus \mathfrak{h}_{0}, \quad \mathrm{~F}_{0}=\mathfrak{h}_{0} .
$$


The Lie superalgebra structure on $F$ satisfies $\left[F_{i}, F_{j}\right] \subset F_{i+j}$ and we are interested in those structures such that the components of the Lie brackets of zero filtration degree coincide with the Lie brackets of $\mathfrak{h}$.

For the Lie superalgebras of interest we can be very concrete and describe the most general filtered deformation of $\mathfrak{h}$ by the following brackets:

$$
\begin{aligned}
{\left[\mathfrak{h}_{0}, \mathrm{~V}\right] } & \subset \mathrm{V} \oplus \mathfrak{h}_{0} \\
{[\mathrm{~S}, \mathrm{~S}] } & \subset \mathrm{V} \oplus \mathfrak{h}_{0} \\
{[\mathrm{~V}, \mathrm{~S}] } & \subset \mathrm{S} \\
{[\mathrm{V}, \mathrm{V}] } & \subset \mathrm{V} \oplus \mathfrak{h}_{0}
\end{aligned}
$$

and the condition that the associated graded Lie superalgebra should be isomorphic to $\mathfrak{h}$ translates into the condition that the component in $\mathrm{V}$ of the brackets $\left[\mathfrak{h}_{0}, \mathrm{~V}\right]$ and $[\mathrm{S}, \mathrm{S}]$ should not be modified from the ones in the Poincaré superalgebra. The components of the Lie brackets of non-zero filtration degree are as follows:

(i) the even component $\mu$ is the sum $\mu=\alpha+\beta+\gamma+\rho$ of the degree-2 maps

$$
\begin{aligned}
& \alpha: \Lambda^{2} \mathrm{~V} \rightarrow \mathrm{V}, \quad \beta: \mathrm{V} \otimes \mathrm{S} \rightarrow \mathrm{S}, \\
& \gamma: \odot^{2} \mathrm{~S} \rightarrow \mathfrak{h}_{0}, \quad \rho: \mathfrak{h}_{0} \otimes \mathrm{V} \rightarrow \mathfrak{h}_{0}
\end{aligned}
$$

(ii) and the even component $\delta: \Lambda^{2} \vee \rightarrow \mathfrak{h}_{0}$ of degree 4 .

Calculating the deformations involves, at first order, the calculation of the cohomology of an appropriate refinement of the Chevalley-Eilenberg complex which we now describe. It is a refinement (by degree) $\mathrm{H}^{\bullet \bullet \bullet}(\mathfrak{m}, \mathfrak{g})$ of the usual Chevalley-Eilenberg cohomology $\mathrm{H}^{\bullet}(\mathfrak{g}, \mathfrak{g})$ associated with a Lie (super)algebra $\mathfrak{g}$ and its adjoint representation to the case of $\mathbb{Z}$-graded Lie (super)algebras $\mathfrak{g}=\bigoplus_{\mathfrak{j} \in \mathbb{Z}} \mathfrak{g}_{j}$ with negatively graded part $\mathfrak{m}=\bigoplus_{\mathfrak{j}<0} \mathfrak{g}_{\mathfrak{j}}$. We will consider first the case of the full Poincaré superalgebra $\mathfrak{g}$.

2.2. The Spencer complex. The cochains of the Spencer complex are even linear maps $\Lambda^{p} \mathfrak{m} \rightarrow \mathfrak{g}$ or, equivalently, even elements of $\mathfrak{g} \otimes \Lambda^{p} \mathfrak{m}^{*}$, where $\Lambda^{\bullet}$ is meant here in the super sense. One extends the degree in $\mathfrak{g}$ to such cochains by declaring that $\mathfrak{g}_{j}^{*}$ has degree $-j$. Since the $\mathbb{Z}$ and $\mathbb{Z}_{2}$ gradings are compatible, even (resp. odd) cochains have even (resp. odd) degree. It is not hard to see that the even $p$-cochains of highest degree are the maps $\Lambda^{p} V \rightarrow \mathfrak{s o}(V)$, which have degree $2 p$. The even $p$-cochains of lowest degree are those in $\operatorname{Hom}\left(\odot^{p} S, V\right)$, for $p \equiv 0(\bmod 2)$, which have degree $p-2$, and those in $\operatorname{Hom}\left(\odot^{p} S, S\right)$ and $\operatorname{Hom}\left(\odot^{p-1} S \otimes V, V\right)$, for $p \equiv 1(\bmod 2)$, which have degree $p-1$. As we will see below, the Spencer differential has degree 0, so the complex breaks up into a direct of sum of finite complexes for each degree.

The spaces in the complexes of even cochains for small degree are given in Table 1; although the complex in degree 4 has cochains also for $p=5,6$ which the table omits. We shall be mainly interested in $p=2$ in this paper, which, as we will see in Theorem 9 later on, corresponds to infinitesimal deformations.

Let $C^{d, p}(\mathfrak{m}, \mathfrak{g})$ denote the space of $p$-cochains of degree $d$. The Spencer differential $\partial: C^{d, p}(\mathfrak{m}, \mathfrak{g}) \rightarrow C^{d, p+1}(\mathfrak{m}, \mathfrak{g})$ is the Chevalley-Eilenberg differential for the Lie superalgebra $\mathfrak{m}$ relative to its module $\mathfrak{g}$ with respect to the adjoint action. For $p=0,1,2$ 
Table 1. Even $p$-cochains of small degree

\begin{tabular}{|c|c|c|c|c|c|}
\hline \multirow[t]{2}{*}{ Deg } & \multicolumn{5}{|l|}{$p$} \\
\hline & 0 & 1 & 2 & 3 & 4 \\
\hline 0 & $\mathfrak{s o}(\mathrm{V})$ & $\begin{array}{l}\mathrm{S} \rightarrow \mathrm{S} \\
\mathrm{V} \rightarrow \mathrm{V}\end{array}$ & $\odot^{2} \mathrm{~S} \rightarrow \mathrm{V}$ & & \\
\hline 2 & & $\mathrm{~V} \rightarrow \mathfrak{s o}(\mathrm{V})$ & $\begin{array}{l}\wedge^{2} \mathrm{~V} \rightarrow \mathrm{V} \\
\mathrm{V} \otimes \mathrm{S} \rightarrow \mathrm{S} \\
\odot^{2} \mathrm{~S} \rightarrow \mathfrak{s o}(\mathrm{V})\end{array}$ & $\begin{array}{l}\odot^{3} S \rightarrow S \\
\odot^{2} S \otimes V \rightarrow V\end{array}$ & $\odot^{4} \mathrm{~S} \rightarrow \mathrm{V}$ \\
\hline 4 & & & $\wedge^{2} \mathrm{~V} \rightarrow \mathfrak{s o}(\mathrm{V})$ & $\begin{array}{l}\odot^{2} S \otimes V \rightarrow \mathfrak{s o}(V) \\
\Lambda^{2} V \otimes S \rightarrow S \\
\Lambda^{3} V \rightarrow V\end{array}$ & $\begin{array}{l}\odot^{4} S \rightarrow \mathfrak{s o}(V) \\
\odot^{3} S \otimes V \rightarrow S \\
\odot^{2} S \otimes \Lambda^{2} V \rightarrow V\end{array}$ \\
\hline
\end{tabular}

and $d \equiv 0(\bmod 2)$ it is explicitly given by the following expressions:

$$
\begin{aligned}
& \partial: C^{d, 0}(\mathfrak{m}, \mathfrak{g}) \rightarrow C^{d, 1}(\mathfrak{m}, \mathfrak{g}) \\
& \partial \varphi(X)=[X, \varphi] \\
& \partial: C^{d, 1}(\mathfrak{m}, \mathfrak{g}) \rightarrow C^{d, 2}(\mathfrak{m}, \mathfrak{g}) \\
& \partial \varphi(X, Y)=[X, \varphi(Y)]-(-1)^{x y}[Y, \varphi(X)]-\varphi([X, Y]), \\
& \partial: C^{d, 2}(\mathfrak{m}, \mathfrak{g}) \rightarrow C^{d, 3}(\mathfrak{m}, \mathfrak{g}) \\
& \partial \varphi(X, Y, Z) \\
& \quad=[X, \varphi(Y, Z)]+(-1)^{x(y+z)}[Y, \varphi(Z, X)]+(-1)^{z(x+y)}[Z, \varphi(X, Y)] \\
& \quad-\varphi([X, Y], Z)-(-1)^{x(y+z)} \varphi([Y, Z], X)-(-1)^{z(x+y)} \varphi([Z, X], Y),
\end{aligned}
$$

where $x, y, \ldots$ denote the parity of elements $X, Y, \ldots$ of $\mathfrak{m}$ and $\varphi \in C^{d, p}(\mathfrak{m}, \mathfrak{g})$ with $p=0,1,2$ respectively.

The space of cochains $C^{d, p}(\mathfrak{m}, \mathfrak{g})$ is an $\mathfrak{s o}(\mathrm{V})$-module and the same is true for the spaces of cocycles and coboundaries, as $\partial$ is $\mathfrak{s o}(\mathrm{V})$-equivariant; this implies that each cohomology group $\mathrm{H}^{\mathrm{d}, \mathfrak{p}}(\mathfrak{m}, \mathfrak{g})$ is an $\mathfrak{s o}(\mathrm{V})$-module, in a natural way. This equivariance is very useful in calculations, as we will have ample opportunity to demonstrate.

Many of the components of the Spencer differential turn out to be injective. For instance, for all $\varphi \in \operatorname{Hom}\left(\Lambda^{2} \vee, \mathfrak{s o}(V)\right)$ in degree 4 , one has

$$
\begin{aligned}
& \partial \varphi\left(s_{1}, s_{2}, v_{1}\right)=-\varphi\left(\left[s_{1}, s_{2}\right], v_{1}\right) \\
& \partial \varphi\left(v_{1}, v_{2}, s_{1}\right)=\left[s_{1}, \varphi\left(v_{1}, v_{2}\right)\right]
\end{aligned}
$$

where $s_{1}, s_{2} \in \mathrm{S}$ and $v_{1}, v_{2} \in \mathrm{V}$ and the two components

$$
\begin{aligned}
& \operatorname{Hom}\left(\Lambda^{2} \mathrm{~V}, \mathfrak{s o}(\mathrm{V})\right) \rightarrow \operatorname{Hom}\left(\odot^{2} \mathrm{~S} \otimes \mathrm{V}, \mathfrak{s o}(\mathrm{V})\right) \\
& \operatorname{Hom}\left(\Lambda^{2} \mathrm{~V}, \mathfrak{s o}(\mathrm{V})\right) \rightarrow \operatorname{Hom}\left(\Lambda^{2} \mathrm{~V} \otimes S, S\right)
\end{aligned}
$$

of $\partial: C^{4,2}(\mathfrak{m}, \mathfrak{g}) \rightarrow C^{4,3}(\mathfrak{m}, \mathfrak{g})$ are injective (in the first case one uses that $\mathfrak{m}$ is fundamental). We also note for completeness that the third component is surjective but has nonzero kernel, giving rise to the short exact sequence

$$
0 \rightarrow \mathrm{V} \boxplus \rightarrow \operatorname{Hom}\left(\Lambda^{2} \vee, \mathfrak{s o}(\mathrm{V})\right) \rightarrow \operatorname{Hom}\left(\Lambda^{3} \vee, \vee\right) \rightarrow 0
$$


where $\mathrm{V} \boxplus$ is the space of algebraic curvature operators; that is, the subspace of $S^{2} \Lambda^{2} \mathrm{~V}$ satisfying the algebraic Bianchi identity. One has the following

Lemma 1. The group $\mathrm{H}^{\mathrm{d}, 2}(\mathfrak{m}, \mathfrak{g})=0$ for all even $\mathrm{d} \geqslant 4$.

Proof. If $\mathrm{d}=4$ then $\left.\operatorname{Ker} \partial\right|_{\mathrm{C}^{4,2}(\mathfrak{m}, \mathfrak{g})}=0$ from the previous observations; if $\mathrm{d}>4$ then the space of cochains $C^{d, 2}(\mathfrak{m}, \mathfrak{g})=0$ and the claim is immediate.

In degree 2 it is convenient to consider the decomposition of $\mathfrak{s o}(\mathrm{V})$-modules

$$
\mathrm{C}^{2,2}(\mathfrak{m}, \mathfrak{g})=\operatorname{Hom}\left(\Lambda^{2} \mathrm{~V}, \mathrm{~V}\right) \oplus \operatorname{Hom}(\mathrm{V} \otimes \mathrm{S}, \mathrm{S}) \oplus \operatorname{Hom}\left(\odot^{2} \mathrm{~S}, \mathfrak{s o}(\mathrm{V})\right)
$$

and the corresponding $\mathfrak{s o}(\mathrm{V})$-equivariant projections

$$
\begin{aligned}
& \pi^{\alpha}: C^{2,2}(\mathfrak{m}, \mathfrak{g}) \rightarrow \operatorname{Hom}\left(\Lambda^{2} \vee, V\right) \\
& \pi^{\beta}: C^{2,2}(\mathfrak{m}, \mathfrak{g}) \rightarrow \operatorname{Hom}(\mathrm{V} \otimes S, S) \text { and } \\
& \pi^{\gamma}: C^{2,2}(\mathfrak{m}, \mathfrak{g}) \rightarrow \operatorname{Hom}\left(\odot^{2} S, \mathfrak{s o}(V)\right) .
\end{aligned}
$$

We find that

$$
\partial \varphi\left(s_{1}, s_{2}\right)=-\varphi\left(\left[s_{1}, s_{2}\right]\right) \text { and } \partial \varphi\left(v_{1}, s_{1}\right)=\left[\varphi\left(v_{1}\right), s_{1}\right]
$$

for all $\varphi \in \operatorname{Hom}(\mathrm{V}, \mathfrak{s o}(\mathrm{V}))$, and that two of the three components of $\partial: \mathrm{C}^{2,1}(\mathfrak{m}, \mathfrak{g}) \rightarrow$ $\mathrm{C}^{2,2}(\mathfrak{m}, \mathfrak{g})$ are injective:

$$
\begin{aligned}
& \operatorname{Hom}(\mathrm{V}, \mathfrak{s o}(\mathrm{V})) \hookrightarrow \operatorname{Hom}\left(\odot^{2} \mathrm{~S}, \mathfrak{s o}(\mathrm{V})\right) \\
& \operatorname{Hom}(\mathrm{V}, \mathfrak{s o}(\mathrm{V})) \hookrightarrow \operatorname{Hom}(\mathrm{V} \otimes \mathrm{S}, \mathrm{S})
\end{aligned}
$$

On the other hand, the image of $\varphi$ under $\partial^{\alpha}$ is given by

$$
\partial^{\alpha} \varphi\left(v_{1}, v_{2}\right)=\left[v_{1}, \varphi\left(v_{2}\right)\right]-\left[v_{2}, \varphi\left(v_{1}\right)\right]
$$

for $v_{1}, v_{2} \in \mathrm{V}$. This easily implies the following

Lemma 2. The component

$$
\partial^{\alpha}:=\pi^{\alpha} \circ \partial: \operatorname{Hom}(\mathrm{V}, \mathfrak{s o}(\mathrm{V})) \rightarrow \operatorname{Hom}\left(\Lambda^{2} \mathrm{~V}, \mathrm{~V}\right)
$$

of the Spencer differential is an isomorphism.

\section{Infinitesimal Deformations}

In this section we first calculate the cohomology group

$$
H^{2,2}(\mathfrak{m}, \mathfrak{g})=\frac{\operatorname{ker} \partial: C^{2,2}(\mathfrak{m}, \mathfrak{g}) \rightarrow C^{2,3}(\mathfrak{m}, \mathfrak{g})}{\partial C^{2,1}(\mathfrak{m}, \mathfrak{g})},
$$

and then consider the $\mathbb{Z}$-graded Lie subalgebras $\mathfrak{h}$ of the Poincare superalgebra. Using the results obtained for $\mathfrak{g}$, we will describe the groups $H^{\mathrm{d}, 2}(\mathfrak{m}, \mathfrak{h})$ for all $d \geqslant 2$ even and then prove Theorem 9 about the infinitesimal deformations of $\mathfrak{h}$. 
3.1. Infinitesimal deformations of $\mathfrak{g}$. We depart from the following observation.

Lemma 3. Every cohomology class $[\alpha+\beta+\gamma] \in \mathrm{H}^{2,2}(\mathfrak{m}, \mathfrak{g})$ with $\alpha \in \operatorname{Hom}\left(\Lambda^{2} \vee, V\right)$, $\beta \in \operatorname{Hom}(\mathrm{V} \otimes \mathrm{S}, \mathrm{S})$ and $\gamma \in \operatorname{Hom}\left(\odot^{2} \mathrm{~S}, \mathfrak{s o}(\mathrm{V})\right)$, has a unique cocycle representative with $\alpha=0$.

Proof. It follows from Lemma 2 that given any $\alpha \in \operatorname{Hom}\left(\Lambda^{2} \vee, V\right)$, there is a unique $\tilde{\alpha} \in C^{2,1}(\mathfrak{m}, \mathfrak{g})$ such that $\partial \tilde{\alpha}=\alpha+\tilde{\beta}+\tilde{\gamma}$, for some $\tilde{\beta} \in \operatorname{Hom}(\mathrm{V} \otimes S, S)$ and some $\tilde{\gamma} \in$ $\operatorname{Hom}\left(\odot^{2} S, \mathfrak{s o}(V)\right)$. Therefore given any cocycle $\alpha+\beta+\gamma$ we may add the coboundary $\partial(-\tilde{\alpha})$ without changing its cohomology class, resulting in the cocycle $(\beta-\tilde{\beta})+(\gamma-\tilde{\gamma})$, which has no component in $\operatorname{Hom}\left(\wedge^{2} \vee, V\right)$.

In other words, $\mathrm{H}^{2,2}(\mathfrak{m}, \mathfrak{g})$ is isomorphic as an $\mathfrak{s o}(\mathrm{V})$-module to the kernel of the Spencer differential restricted to $\operatorname{Hom}(V \otimes S, S) \oplus \operatorname{Hom}\left(\odot^{2} S, \mathfrak{s o}(V)\right)$. It follows from Eq. (8) for the Spencer differential, that a cochain $\beta+\gamma \in \operatorname{Hom}(V \otimes S, S) \oplus \operatorname{Hom}\left(\odot^{2} S, \mathfrak{s o}(V)\right)$ is a cocycle if and only if the following pair of "cocycle conditions" are satisfied:

$$
[\gamma(\mathrm{s}, \mathrm{s}), v]=-2[\mathrm{~s}, \beta(\nu, s)] \quad \forall \mathrm{s} \in \mathrm{S}, \nu \in \mathrm{V},
$$

and

$$
[\gamma(s, s), s]=-\beta([s, s], s) \quad \forall s \in S .
$$

We note that the cocycle condition (10) fully expresses $\gamma$ in terms of $\beta$, once the fact that $\gamma$ takes values into $\mathfrak{s o}(\mathrm{V})$ has been taken into account. To this aim, we define for any $v \in V$ the endomorphism $\beta_{v} \in \operatorname{End}(S)$ by $\beta_{v}(s)=\beta(v, s)$ and rewrite (10) as

$$
[\gamma(\mathrm{s}, \mathrm{s}), v]=-2\left[\mathrm{~s}, \beta_{v}(\mathrm{~s})\right] .
$$

Take the inner product with $v$ and use Eqs. (4) and (3) to arrive at

$$
0=2\left\langle s, v \cdot \beta_{v}(s)\right\rangle \quad \forall s \in S, v \in \mathrm{V} .
$$

This says that for all $v \in V$ the endomorphism $v \cdot \beta_{v}$ of $S$ is symmetric relative to the symplectic form $\langle-,-\rangle$. Equivalently, it is fixed by the anti-involution $\sigma$ defined by the symplectic form: $\sigma\left(v \cdot \beta_{v}\right)=v \cdot \beta_{v}$.

We now observe that if $\Theta \in \mathrm{C} \ell(\mathrm{V})$ is fixed by $\sigma$, then so are $v \cdot \Theta \cdot v$ and (trivially) $v \cdot v \cdot \Theta=-\eta(v, v) \Theta$, so that we have an immediate class of solutions to Eq. (12): namely, $\beta_{v}=v \cdot \Theta+\Theta^{\prime} \cdot v$, where $\Theta, \Theta^{\prime} \in \mathrm{Cl}(\mathrm{V})$ are fixed by $\sigma$.

Following our conventions on Clifford algebras and spinors in Appendix A, we have

$$
\operatorname{End}(S) \cong \bigoplus_{p=0}^{5} \wedge^{p} V
$$

as $\mathfrak{s o}(\mathrm{V})$-modules. The anti-involution $\sigma$ preserves each submodule and acts on the submodule of type $\Lambda^{p} \vee$ as $(-1)^{p(p+1) / 2} \mathbb{1}$, so that the submodule fixed by $\sigma$ correspond to $\Lambda^{2} S \cong \Lambda^{0} V \oplus \Lambda^{3} V \oplus \Lambda^{4} V$. In other words, Eq. (12) says that for all $v \in V$,

$$
v \cdot \beta_{v} \in \Lambda^{0} \mathrm{~V} \oplus \Lambda^{3} \mathrm{~V} \oplus \Lambda^{4} \mathrm{~V}
$$

that is, strictly speaking, in the image of $\Lambda^{0} \vee \oplus \Lambda^{3} \vee \oplus \Lambda^{4} V$ in $\operatorname{End}(S)$.

As we have seen above, we can exhibit solutions to Eq. (14) of the form

$$
\beta_{v}=v \cdot \Theta+\Theta^{\prime} \cdot v
$$

for $\Theta, \Theta^{\prime} \in \Lambda^{0} \vee \oplus \Lambda^{3} \vee \oplus \Lambda^{4} \vee$. Remarkably, it turns out that these are all the solutions to Eq. (14). 
Proposition 4. The general solution of Eq. (14) is

$$
\beta_{v}=\theta_{0} v+v \cdot \theta_{3}+\theta_{3}^{\prime} \cdot v+v \cdot \theta_{4}+\theta_{4}^{\prime} \cdot v
$$

where $\theta_{0} \in \Lambda^{0} \mathrm{~V}, \theta_{3}, \theta_{3}^{\prime} \in \Lambda^{3} \mathrm{~V}$ and $\theta_{4}, \theta_{4}^{\prime} \in \Lambda^{4} \mathrm{~V}$.

Although a more combinatorial proof of Proposition 4 is also possible, we give here a proof which uses representation theory and the $\mathfrak{s o}(\mathrm{V})$-equivariance of the condition (14). To do so, we will use freely the notation in Appendix B and identify the $\mathfrak{s o}(\mathrm{V})$-modules $\operatorname{Hom}(\mathrm{V} \otimes \mathrm{S}, \mathrm{S})$ and $\operatorname{Hom}(\mathrm{V}, \operatorname{End}(\mathrm{S}))$.

We start by reformulating slightly Proposition 4. Let

$$
\Phi: \operatorname{Hom}(\mathrm{V}, \operatorname{End}(\mathrm{S})) \rightarrow \operatorname{Hom}\left(\odot^{2} \mathrm{~V}, \operatorname{End}(\mathrm{S})\right)
$$

denote the $\mathfrak{s o}(V)$-equivariant map which sends $\beta \in \operatorname{Hom}(V, \operatorname{End}(S))$ to $\Phi(\beta)$, given for all $v, w \in V$ by

$$
\Phi(\beta)(v, w)=v \cdot \beta_{w}+w \cdot \beta_{v},
$$

where - stands, as usual, for the Clifford product. We start with a useful observation.

Lemma 5. The map $\Phi: \operatorname{Hom}(\mathrm{V}, \operatorname{End}(\mathrm{S})) \rightarrow \operatorname{Hom}\left(\odot^{2} \mathrm{~V}, \operatorname{End}(\mathrm{S})\right)$ is injective.

Proof. Suppose that $\Phi(\beta)=0$. This means that for all $v \in \mathrm{V}, v \cdot \beta_{v}=0$. By Cliffordmultiplying on the left with $v$, we learn that $\beta_{v}=0$ for all $v \in V$ with $\eta(v, v) \neq 0$. But $v \mapsto \beta_{v}$ is linear and there exists a basis for $V$ whose elements have nonzero norm, hence $\beta_{v}=0$ for all $\nu \in V$ and hence $\beta=0$.

Using the $\mathfrak{s o}(\mathrm{V})$-module decomposition (13)

$$
\operatorname{Hom}(\mathrm{V}, \operatorname{End}(\mathrm{S})) \cong \bigoplus_{\mathrm{p}=0}^{5} \operatorname{Hom}\left(\mathrm{V}, \Lambda^{\mathrm{p}} \mathrm{V}\right),
$$

we may decompose $\beta=\beta_{0}+\beta_{1}+\cdots+\beta_{5}$, where $\beta_{p}$ belongs to the $\mathfrak{s o}(V)$-submodule $\operatorname{Hom}\left(\mathrm{V}, \wedge^{\mathrm{p}} \mathrm{V}\right)$ of $\operatorname{Hom}(\mathrm{V}, \operatorname{End}(\mathrm{S}))$. Similarly we have an $\mathfrak{s o}(\mathrm{V})$-equivariant isomorphism

$$
\operatorname{Hom}\left(\odot^{2} \mathrm{~V}, \operatorname{End}(S)\right) \cong \bigoplus_{\mathrm{q}=0}^{5} \operatorname{Hom}\left(\odot^{2} \mathrm{~V}, \wedge^{\mathrm{q}} \mathrm{V}\right)
$$

and a corresponding decomposition $\theta=\theta_{0}+\theta_{1}+\cdots+\theta_{5}$ of $\theta \in \operatorname{Hom}\left(\odot^{2} \mathrm{~V}\right.$, $\left.\operatorname{End}(S)\right)$, with $\theta_{\mathrm{q}}$ belonging to the $\mathfrak{s o}(\mathrm{V})$-submodule $\operatorname{Hom}\left(\odot^{2} \mathrm{~V}, \Lambda^{\mathrm{q}} \mathrm{V}\right)$ of $\operatorname{Hom}\left(\odot^{2} \mathrm{~V}, \operatorname{End}(\mathrm{S})\right)$.

We now observe that Eq. (14) for $\beta$, which says that $\Phi(\beta)(\nu, w)$ is a symmetric endomorphism of $S$ for all $v, w \in V$, is equivalent to $\Phi(\beta)_{\mathrm{q}}=0$ for $\mathrm{q}=1,2,5$ and recall that the solution space of these three equations contains a submodule of type

$$
\Lambda^{0} \mathrm{~V} \oplus 2 \Lambda^{3} \mathrm{~V} \oplus 2 \Lambda^{4} \mathrm{~V}
$$

From Table 2, which lists the decomposition of $\operatorname{Hom}\left(V, \wedge^{p} V\right)$, for $p=0,1, \ldots, 5$, into irreducible $\mathfrak{s o}(\mathrm{V})$-modules, we see that there is a unique $\mathfrak{s o}(\mathrm{V})$-submodule isomorphic to (16), whose irreducible components appear inside boxes. As explained in Appendix B, the notation $\left(\mathrm{V^{p }} \otimes \wedge^{\mathrm{p}}\right)_{0}$ stands for the kernel of Clifford multiplication.

It follows from this discussion that Proposition 4 is equivalent to the following. 
Table 2. Irreducible components of $\operatorname{Hom}\left(\vee, \wedge^{p} \vee\right)$ for $p=0, \ldots, 5$

\begin{tabular}{ll}
\hline $\mathrm{p}$ & $\operatorname{Hom}\left(\mathrm{V}, \wedge^{\mathrm{p}} \mathrm{V}\right)$ \\
\hline 0 & $\mathrm{~V}$ \\
1 & $\wedge^{0} \mathrm{~V} \oplus \wedge^{2} \mathrm{~V} \oplus \odot_{0}^{2} \mathrm{~V}$ \\
2 & $\mathrm{~V} \oplus \wedge^{3} \mathrm{~V} \oplus\left(\mathrm{V} \otimes \wedge^{2} \mathrm{~V}\right)_{0}$ \\
3 & $\Lambda^{2} \mathrm{~V} \oplus \wedge^{4} \mathrm{~V} \oplus\left(\mathrm{V} \otimes \wedge^{3} \mathrm{~V}\right)_{0}$ \\
4 & $\Lambda^{3} \mathrm{~V} \oplus \wedge^{5} \mathrm{~V} \oplus\left(\mathrm{V} \otimes \wedge^{4} \mathrm{~V}\right)_{0}$ \\
5 & $\Lambda^{4} \mathrm{~V} \oplus \wedge^{5} \mathrm{~V} \oplus\left(\mathrm{V} \otimes \wedge^{5} \mathrm{~V}\right)_{0}$ \\
\hline
\end{tabular}

Table 3. Some irreducible components of $\operatorname{Hom}\left(\odot^{2} \mathrm{~V}, \wedge^{\mathrm{q}} \mathrm{V}\right)$ for $\mathrm{q}=0, \ldots, 5$

\begin{tabular}{ll}
\hline $\mathrm{q}$ & $\operatorname{Hom}\left(\odot^{2} \mathrm{~V}, \wedge^{\mathrm{q}} \mathrm{V}\right)$ \\
\hline 0 & $\wedge^{0} \mathrm{~V} \oplus \odot_{0}^{2} \mathrm{~V}$ \\
1 & $2 \mathrm{~V} \oplus\left(\mathrm{V} \otimes \wedge^{2} \mathrm{~V}\right)_{0}$ \\
2 & $2 \wedge^{2} \mathrm{~V} \oplus \odot{ }_{0}^{2} \mathrm{~V} \oplus\left(\mathrm{V} \otimes \wedge^{3} \mathrm{~V}\right)_{0}$ \\
3 & $2 \Lambda^{3} \mathrm{~V} \oplus\left(\mathrm{V} \otimes \wedge^{4} \mathrm{~V}\right)_{0} \oplus\left(\mathrm{V} \otimes \wedge^{2} \mathrm{~V}\right)_{0}$ \\
4 & $2 \Lambda^{4} \mathrm{~V} \oplus\left(\mathrm{V} \otimes \wedge^{5} \mathrm{~V}\right)_{0} \oplus\left(\mathrm{V} \otimes \wedge^{3} \mathrm{~V}\right)_{0}$ \\
5 & $2 \Lambda^{5} \mathrm{~V} \oplus\left(\mathrm{V} \otimes \wedge^{5} \mathrm{~V}\right)_{0} \oplus\left(\mathrm{V} \otimes \Lambda^{4} \mathrm{~V}\right)_{0}$ \\
\hline
\end{tabular}

Proposition 6. The solution space of Eq. (14) is the unique submodule of $\mathrm{Hom}(\mathrm{V}, \operatorname{End}(\mathrm{S}))$ isomorphic to (16).

Proof. It follows from the first formula in (45) that Clifford multiplication maps $\vee \otimes$ $\Lambda^{\mathfrak{p}} V \rightarrow \Lambda^{\mathfrak{p}-1} \vee \oplus \Lambda^{\mathfrak{p}+1} V$. From this fact and the very definition (15) of the map $\Phi$, it is clear that $\Phi\left(\beta_{p}\right)_{q}=0$ unless $q=p \pm 1$. Therefore Eq. (14) is equivalent to the following system of linear equations:

$$
\begin{aligned}
& \Phi(\beta)_{1}=0 \Longleftrightarrow \Phi\left(\beta_{0}\right)_{1}+\Phi\left(\beta_{2}\right)_{1}=0 \\
& \Phi(\beta)_{2}=0 \Longleftrightarrow \Phi\left(\beta_{1}\right)_{2}+\Phi\left(\beta_{3}\right)_{2}=0 \\
& \Phi(\beta)_{5}=0 \Longleftrightarrow \Phi\left(\beta_{4}\right)_{5}+\Phi\left(\beta_{5}\right)_{5}=0 .
\end{aligned}
$$

Note that each component $\beta_{0}, \ldots, \beta_{5}$ of $\beta$ appear in one and only one of the above three equations. Table 3 lists the irreducible $\mathfrak{s o}(\mathrm{V})$-components in $\operatorname{Hom}\left(\odot^{2} \mathrm{~V}, \wedge^{\mathrm{q}} \mathrm{V}\right)$ for $\mathrm{q}=0, \ldots, 5$ which are isomorphic to one of the irreducible modules appearing in Table 2. By $\mathfrak{s o}(\mathrm{V})$-equivariance the image of $\Phi$ is isomorphic to the direct sum of the irreducible modules displayed in Table 2 and is contained in the direct sum of the irreducible modules displayed in Table 3.

Let $\Phi_{\mathrm{q}}^{\mathrm{p}}$ denote the component of $\Phi$ mapping $\operatorname{Hom}\left(\mathrm{V}, \wedge^{\mathrm{p}} \vee\right)$ to $\operatorname{Hom}\left(\odot^{2} \mathrm{~V}, \wedge^{\mathrm{q}} \mathrm{V}\right)$. We will have proved the proposition if we show that the undesirable irreducible components of $\operatorname{Hom}(V, \operatorname{End}(S))$ (those not boxed in Table 2) are not in the kernel of $\Phi_{q}^{\bullet}$ for $q=1,2,5$. Since each $\Phi_{\mathfrak{q}}^{p}$ is $\mathfrak{s o}(V)$-equivariant, it is enough to show that this is the case for each type of undesirable submodule. We now go through each such submodule in turn.

Let $2 \mathrm{~V}$ be the isotypical component of $\mathrm{V}$ inside $\operatorname{Hom}(\mathrm{V}$, End $(\mathrm{S}))$; it is contained in $\operatorname{Hom}\left(\mathrm{V}, \wedge^{0} \mathrm{~V}\right) \oplus \operatorname{Hom}\left(\mathrm{V}, \wedge^{2} \mathrm{~V}\right)$. From Table 3 and Lemma 5, $\Phi$ maps $2 \mathrm{~V}$ injectively 
into $\operatorname{Hom}\left(\odot^{2} \mathrm{~V}, \wedge^{1} \mathrm{~V}\right)$. It follows that $\left.\Phi\right|_{2} \mathrm{~V}=\left.\left(\Phi_{1}^{0}+\Phi_{1}^{2}\right)\right|_{2 \mathrm{~V}}: 2 \mathrm{~V} \rightarrow \operatorname{Hom}\left(\odot^{2} \mathrm{~V}, \wedge^{1} \mathrm{~V}\right)$ is injective, and thus the first equation in (17) is not satisfied by any nonzero $\beta=\beta_{0}+\beta_{2} \in$ $2 \mathrm{~V}$ and the solution space of Eq. (14) does not contain any submodule isomorphic to $\mathrm{V}$.

A similar argument shows that the isotypical components $2 \Lambda^{2} V$ and $2 \Lambda^{5} V$ are mapped injectively to submodules of $\operatorname{Hom}\left(\odot^{2} \mathrm{~V}, \Lambda^{2} \mathrm{~V}\right)$ and $\operatorname{Hom}\left(\odot^{2} \mathrm{~V}, \Lambda^{5} \mathrm{~V}\right)$, respectively, and hence the solution space of Eq. (14) does not contain any submodule isomorphic to $\wedge^{2} V$ or $\wedge^{5} \vee$ either.

The remaining submodules are isomorphic to $\left(\mathrm{V} \otimes \Lambda^{\mathrm{p}} \mathrm{V}\right)_{0}$ for $\mathrm{p}=1,2,3,4,5$ and are unique. Since any such module is irreducible, the equivariant map $\Phi$ is either zero or an isomorphism when restricted to it. Thus we find it easiest to pick a nonzero element and show that its image under the relevant component of $\Phi$ is nonzero.

Let $\beta=\mathbf{e}_{1}^{b} \otimes \mathbf{e}_{2}+\mathbf{e}_{2}^{b} \otimes \mathbf{e}_{1}$. It belongs to the submodule of type $(\mathrm{V} \otimes \mathrm{V})_{0} \cong \odot_{0}^{2} \mathrm{~V}$ and a calculation in $\mathrm{C} \ell(\mathrm{V})$ shows that

$$
\Phi(\beta)_{2}\left(e_{1}, e_{1}\right)=-2 e_{1} \wedge e_{2} \neq 0 .
$$

Let $\beta=e_{1}^{b} \otimes e_{2} \wedge e_{3}+e_{2}^{b} \otimes e_{1} \wedge e_{3}$. It belongs to the submodule of type $\left(V \otimes \wedge^{2} V\right)_{0}$ and

$$
\Phi(\beta)_{1}\left(\mathbf{e}_{1}+\mathbf{e}_{2}, \mathbf{e}_{1}+\mathbf{e}_{2}\right)=-4 \mathbf{e}_{3} \neq 0 .
$$

Let $\beta=e_{1}^{b} \otimes e_{2} \wedge e_{3} \wedge e_{4}+e_{2}^{b} \otimes e_{1} \wedge e_{3} \wedge e_{4}$. It belongs to the submodule of type $\left(\mathrm{V} \otimes \wedge^{3} \mathrm{~V}\right)_{0}$ and

$$
\Phi(\beta)_{2}\left(e_{1}+e_{2}, e_{1}+e_{2}\right)=-4 e_{3} \wedge e_{4} \neq 0 .
$$

Let $\beta=e_{1}^{b} \otimes e_{2} \wedge e_{3} \wedge e_{4} \wedge e_{5}+e_{2}^{b} \otimes e_{1} \wedge e_{3} \wedge e_{4} \wedge e_{5}$. It belongs to the submodule of type $\left(\mathrm{V} \otimes \Lambda^{4} \mathrm{~V}\right)_{0}$ and

$$
\Phi(\beta)_{5}\left(e_{1}, e_{1}\right)=-2 e_{1} \wedge e_{2} \wedge e_{3} \wedge e_{4} \wedge e_{5} \neq 0 .
$$

Finally, let $\beta=e_{1}^{b} \otimes e_{2} \wedge e_{3} \wedge e_{4} \wedge e_{5} \wedge e_{6}+e_{2}^{b} \otimes e_{1} \wedge e_{3} \wedge e_{4} \wedge e_{5} \wedge e_{6}$. It belongs to the submodule of type $\left(\mathrm{V} \otimes \Lambda^{5} \mathrm{~V}\right)_{0}$ and

$$
\Phi(\beta)_{5}\left(e_{1}, e_{1}\right)=-2 e_{1} \wedge e_{2} \wedge e_{3} \wedge e_{4} \wedge e_{5} \wedge e_{6} \neq 0,
$$

thought as an element of $\Lambda^{6} V \simeq \Lambda^{5} \vee$ in $\operatorname{End}(S)$. This concludes the proof of the proposition.

Returning to the cocycle conditions (10) and (11), we now observe that the first one simply defines $\gamma$ in terms of $\beta$, which is then subject to the second condition. Given the general form of $\beta_{v}$ found in Proposition 4, we solve the cocycle conditions in the following

Proposition 7. The general solution $(\beta, \gamma)$ of the cocycle conditions (10) and (11) is of the form $(\beta, \gamma)=\left(\beta^{\varphi}, \gamma^{\varphi}\right)$ for a unique $\varphi \in \Lambda^{4} \mathrm{~V}$ such that

$$
\begin{aligned}
\beta_{v}^{\varphi}(s) & =v \cdot \varphi \cdot s-3 \varphi \cdot v \cdot s, \\
{\left[\gamma^{\varphi}(s, s), v\right] } & =-2\left[s, \beta_{v}^{\varphi}(s)\right] \\
& =-2[s, v \cdot \varphi \cdot s]+6[s, \varphi \cdot v \cdot s],
\end{aligned}
$$

for all $v \in \mathrm{V}$ and $\mathrm{s} \in \mathrm{S}$. In particular $\mathrm{H}^{2,2}(\mathfrak{m}, \mathfrak{g}) \simeq \wedge^{4} \mathrm{~V}$ as an $\mathfrak{s o}(\mathrm{V})$-module. 
Remark. Expanding the Clifford products, we may rewrite $\beta_{v}^{\varphi}$ as

$$
\beta_{v}^{\varphi}=-2 v \wedge \varphi-4 \iota_{v} \varphi,
$$

which agrees with the zeroth order terms in the connection D in Eq. (1) for $\varphi=\frac{1}{24} \mathrm{~F}$. The connection D encodes the geometry of (supersymmetric) bosonic backgrounds of 11-dimensional supergravity: not just does it define the notion of a Killing spinor, but its curvature encodes the bosonic field equations. Indeed, as shown in [6], the field equations are precisely the vanishing of the gamma-trace of the curvature of D. Proposition 7 can be paraphrased as showing that we are able to reconstruct 11-dimensional supergravity (at least at the level of the bosonic field equations) from the Spencer cohomology of the Poincaré superalgebra.

Proof. Rewriting Eq. (11) as

$$
[\gamma(s, s), s]+\beta_{[s, s]}(s)=0,
$$

with $\gamma$ given in terms of $\beta$ by Eq. (10), we see that its solutions $\beta$ are the kernel of an $\mathfrak{s o}(V)$-equivariant linear map. The kernel consists of submodules and hence it is enough, given Proposition 6 , to study this equation separately for $\beta$ belonging to an isotypical component of type $\Lambda^{0} \mathrm{~V}, 2 \Lambda^{3} \mathrm{~V}$ and $2 \Lambda^{4} \mathrm{~V}$, respectively.

It is convenient in what follows to work in $\mathrm{C} \ell(\mathrm{V})$. This uses the notation explained in Appendix B and the Einstein summation convention.

Let us define $\gamma(s, s)^{\mu}{ }_{v}$ by

$$
\gamma(\mathrm{s}, \mathrm{s})\left(\boldsymbol{e}_{v}\right)=\gamma(\mathrm{s}, \mathrm{s})^{\mu}{ }_{\nu} \boldsymbol{e}_{\mu} .
$$

It follows from the first cocycle condition (10) that

$$
\gamma(s, s)_{\mu \nu}=2 \bar{s} \Gamma_{\mu} \beta_{\nu} s,
$$

where have abbreviated $\beta_{\boldsymbol{e}_{v}}$ by $\beta_{v}$. Using Eq. (40), the image of $\gamma(s, s)$ in $C \ell(V)$ is given by

$$
\gamma(\mathrm{s}, \mathrm{s}) \mapsto-\frac{1}{2}\left(\bar{s} \Gamma_{\mu} \beta_{\nu} s\right) \Gamma^{\mu \nu}
$$

and hence

$$
[\gamma(s, s), s]=-\frac{1}{2}\left(\bar{s} \Gamma_{\mu} \beta_{\nu} s\right) \Gamma^{\mu \nu} s .
$$

The second term of the second cocycle condition (11) is given by

$$
\beta_{[s, s]} s=-\left(\bar{s} \Gamma^{\mu} s\right) \beta_{\mu} s,
$$

so that the second cocycle condition becomes

$$
\frac{1}{2}\left(\bar{s} \Gamma_{\mu} \beta_{\nu} s\right) \Gamma^{\mu \nu} s+\left(\bar{s} \Gamma^{\mu} s\right) \beta_{\mu} s=0 .
$$

It is enough to consider three different cases for $\beta$.

Let $\beta$ be of type $\Lambda^{0} V$, so that $\beta_{\mu}=\theta_{0} \Gamma_{\mu}$ for some $\theta_{0} \in \Lambda^{0} V$. Then Eq. (20) becomes

$$
\frac{1}{2} \theta_{0}\left(\bar{s} \Gamma^{\mu \nu} s\right) \Gamma_{\mu \nu} s=-\theta_{0}\left(\bar{s} \Gamma^{\mu} s\right) \Gamma_{\mu} s .
$$

From the first of Eqs. (47), (21) becomes

$$
\theta_{0}\left(\bar{s} \Gamma^{\mu} s\right) \Gamma_{\mu} s=0 .
$$


Taking the symplectic inner product with s we find

$$
\theta_{0}\left(\bar{s} \Gamma^{\mu} s\right)\left(\bar{s} \Gamma_{\mu} s\right)=\theta_{0} \eta([s, s],[s, s])=0
$$

for all $s \in S$, where $[s, s]$ is the Dirac current of $s$. On the other hand there always exists an $s$ for which $\eta([s, s],[s, s])>0$ and hence the only way (21) is satisfied for all $s$ is if $\theta_{0}=0$.

The next two cases, $\beta$ in $2 \Lambda^{3} \mathrm{~V}$ and $2 \Lambda^{4} \mathrm{~V}$, are computationally more involved. It pays to exploit the equivariance under $\mathfrak{s o}(\mathrm{V})$, which implies first of all that the solution space is an $\mathfrak{s o}(\mathrm{V})$-module. We also notice that both $\Lambda^{3} \mathrm{~V}$ and $\Lambda^{4} \mathrm{~V}$ are real irreducible representations of $\mathfrak{s o}(\mathrm{V})$ which remain irreducible upon complexification. This means that the only $\mathfrak{s o}(\mathrm{V})$-equivariant endomorphisms of $\Lambda^{3} V$ and $\Lambda^{4} \mathrm{~V}$ are real multiples of the identity.

Now suppose that $\beta$ is in $2 \Lambda^{3} \vee$. The solution space to Eq. (20) is an $\mathfrak{s o}(V)$-submodule of $\Lambda^{3} V \oplus \Lambda^{3} V$, hence it is either all of $2 \Lambda^{3} V$ (which happens if the equations are identically zero), or a copy of $\Lambda^{3} \vee$ given by the image of

$$
\Lambda^{3} \vee \ni \psi \mapsto\left(t_{1} \psi, t_{2} \psi\right) \in \Lambda^{3} \vee \oplus \Lambda^{3} V
$$

for some $t_{1}, t_{2} \in \mathbb{R}$. We also allow for the case of a zero-dimensional solution space when $t_{1}=t_{2}=0$. We put $\beta_{\mu}=t_{1} \Gamma_{\mu} \psi+t_{2} \psi \Gamma_{\mu}$, for $\psi \in \Lambda^{3} V$, into Eq. (20) to arrive at the following set of equations for $t_{1}, t_{2} \in \mathbb{R}$ :

$$
0=t_{1}\left(\frac{1}{2}\left(\bar{s} \Gamma_{\mu \nu} \psi s\right) \Gamma^{\mu \nu} s+\left(\bar{s} \Gamma^{\mu} s\right) \Gamma_{\mu} \psi s\right)+t_{2}\left(\frac{1}{2}\left(\bar{s} \Gamma_{\mu} \psi \Gamma_{v} s\right) \Gamma^{\mu \nu} s+\left(\bar{s} \Gamma^{\mu} s\right) \psi \Gamma_{\mu} s\right)
$$

parametrised by all $s \in S$ and all $\psi \in \Lambda^{3} V$. It is simply a matter of choosing $s$ and $\psi$ and calculating the resulting expression using our favourite explicit realisations of the Clifford algebra to obtain equations for $t_{1}$ and $t_{2}$. We omit the details, but simply record that the only solution is $t_{1}=t_{2}=0$, so that there is no component of the solution space of Eq. (20) of type $\wedge^{3} \mathrm{~V}$.

Finally, let $\beta$ be in $2 \Lambda^{4} \mathrm{~V}$. As before, the solution space is an $\mathfrak{s o}(\mathrm{V})$-submodule of $\Lambda^{4} \mathrm{~V} \oplus \Lambda^{4} \mathrm{~V}$, whence it is either all of $2 \Lambda^{4} \mathrm{~V}$, or else given by the image of

$$
\Lambda^{4} \vee \ni \phi \mapsto\left(t_{1} \phi, t_{2} \phi\right) \in \Lambda^{4} V \oplus \Lambda^{4} V
$$

for some $t_{1}, t_{2} \in \mathbb{R}$ and again we allow for the case of a zero-dimensional solution space when $t_{1}=t_{2}=0$. Putting $\beta_{\mu}=t_{1} \Gamma_{\mu} \phi+t_{2} \phi \Gamma_{\mu}$, for $\phi \in \Lambda^{4} V$, into Eq. (20) we arrive at the following set of equations for $t_{1}, t_{2} \in \mathbb{R}$ :

$$
0=t_{1}\left(\frac{1}{2}\left(\bar{s} \Gamma_{\mu \nu} \phi s\right) \Gamma^{\mu \nu} s+\left(\bar{s} \Gamma^{\mu} s\right) \Gamma_{\mu} \phi s\right)+t_{2}\left(\frac{1}{2}\left(\bar{s} \Gamma_{\mu} \phi \Gamma_{v} s\right) \Gamma^{\mu v} s+\left(\bar{s} \Gamma^{\mu} s\right) \phi \Gamma_{\mu} s\right)
$$

parametrised by all $s \in S$ and all $\phi \in \Lambda^{4} \mathrm{~V}$. A further simplification due to $\mathfrak{s o}(\mathrm{V})$ equivariance is the following. Since the equations are homogeneous in $s$, we need only take $s$ from a set consisting of a representative of each projectivised orbit of $\operatorname{Spin}(\mathrm{V})$ on $S \backslash\{0\}$. As shown, for example, in [39], there are two such projectivised orbits, distinguished by the causal character of the associated Dirac current: either null or timelike. Therefore we need only consider two such s: one in each type of orbit. Again we omit the actual details of this calculation and simply record the results: taking the null orbit, 
Table 4. Even $p$-cochains of small degree of $\mathfrak{h} \subset \mathfrak{g}$

\begin{tabular}{|c|c|c|c|c|c|}
\hline \multirow[t]{2}{*}{ Deg } & \multicolumn{5}{|l|}{$\underline{p}$} \\
\hline & 0 & 1 & 2 & 3 & 4 \\
\hline 0 & $\mathfrak{h}_{0}$ & $\begin{array}{l}\mathrm{S} \rightarrow \mathrm{S} \\
\mathrm{V} \rightarrow \mathrm{V}\end{array}$ & $\odot^{2} \mathrm{~S} \rightarrow \mathrm{V}$ & & \\
\hline 2 & & $\mathrm{~V} \rightarrow \mathfrak{h}_{0}$ & $\begin{array}{l}\wedge^{2} \mathrm{~V} \rightarrow \mathrm{V} \\
\mathrm{V} \otimes \mathrm{S} \rightarrow \mathrm{S} \\
\odot^{2} \mathrm{~S} \rightarrow \mathfrak{h}_{0}\end{array}$ & $\begin{array}{l}\odot^{3} S \rightarrow S \\
\odot^{2} S \otimes V \rightarrow V\end{array}$ & $\odot^{4} \mathrm{~S} \rightarrow \mathrm{V}$ \\
\hline 4 & & & $\wedge^{2} \mathrm{~V} \rightarrow \mathfrak{h}_{0}$ & $\begin{array}{l}\odot^{2} S \otimes V \rightarrow \mathfrak{h}_{0} \\
\Lambda^{2} \mathrm{~V} \otimes S \rightarrow S \\
\Lambda^{3} \mathrm{~V} \rightarrow \mathrm{V}\end{array}$ & $\begin{array}{l}\odot^{4} S \rightarrow \mathfrak{h}_{0} \\
\odot^{3} S \otimes V \rightarrow S \\
\odot^{2} S \otimes \Lambda^{2} V \rightarrow V\end{array}$ \\
\hline
\end{tabular}

we find that the only equation is $t_{2}=-3 t_{1}$, and imposing this, the equation from the timelike orbit is automatically satisfied.

In summary, the solution of Eq. (20) is

$$
\beta_{\mu}=\Gamma_{\mu} \varphi-3 \varphi \Gamma_{\mu},
$$

for some $\varphi \in \Lambda^{4} \mathrm{~V}$, with the expression for $\gamma$ then following from Eq. (10).

It should be remarked that Eqs. (22) and (23) can also be solved without recourse to an explicit matrix realisation of the Clifford algebra by repeated use of the Fierz identity (46).

We have thus computed the cohomology groups $H^{d, 2}(\mathfrak{m}, \mathfrak{g})$ for all $d \geqslant 2$ even. If $d \geqslant 4$ they are all trivial by Lemma 1 whereas $H^{2,2}(\mathfrak{m}, \mathfrak{g}) \cong \Lambda^{4} \vee$ by Proposition 7 . To prove our first main result on infinitesimal deformations of $\mathfrak{g}$ and its $\mathbb{Z}$-graded subalgebras $\mathfrak{h}$ we also need to determine the analogous groups $H^{d, 2}(\mathfrak{m}, \mathfrak{h})$ for $\mathfrak{h}$, for all $d \geqslant 2$ even.

3.2. Infinitesimal deformations of subalgebras $\mathfrak{h} \subset \mathfrak{g}$. Let $\mathfrak{h}=\mathfrak{h}_{-2} \oplus \mathfrak{h}_{-1} \oplus \mathfrak{h}_{0}$ be a $\mathbb{Z}$-graded subalgebra of the Poincaré superalgebra $\mathfrak{g}$ which differs only in zero degree, that is $\mathfrak{h}_{0} \subset \mathfrak{g}_{0}$ and $\mathfrak{h}_{\mathfrak{j}}=\mathfrak{g}_{\mathfrak{j}}$ for all $\mathfrak{j}<0$. In this section we first calculate the cohomology

$$
\mathrm{H}^{\mathrm{d}, 2}(\mathfrak{m}, \mathfrak{h})=\frac{\operatorname{ker} \partial: \mathrm{C}^{\mathrm{d}, 2}(\mathfrak{m}, \mathfrak{h}) \rightarrow \mathrm{C}^{\mathrm{d}, 3}(\mathfrak{m}, \mathfrak{h})}{\partial \mathrm{C}^{\mathrm{d}, 1}(\mathfrak{m}, \mathfrak{h})}
$$

for all even $d>0$ and then prove Theorem 9 on the filtered deformations of $\mathfrak{h}$. The even $p$-cochains of small degree associated to $\mathfrak{h}$ are displayed in Table 4.

Proposition 8. The group $\mathrm{H}^{\mathrm{d}, 2}(\mathfrak{m}, \mathfrak{h})=0$ for all even $\mathrm{d} \geqslant 4$, whereas

$$
H^{2,2}(\mathfrak{m}, \mathfrak{h})=\frac{\left\{\beta^{\varphi}+\gamma^{\varphi}+\partial \tilde{\alpha} \mid \varphi \in \Lambda^{4} V, \tilde{\alpha}: V \rightarrow \mathfrak{s o}(V) \text { with } \gamma^{\varphi}(s, s)-\tilde{\alpha}([s, s]) \in \mathfrak{h}_{0}\right\}}{\left\{\partial \tilde{\alpha} \mid \tilde{\alpha}: V \rightarrow \mathfrak{h}_{0}\right\}},
$$

where $\left(\beta^{\varphi}, \gamma^{\varphi}\right)$ are as in Proposition 7. In particular any cohomology class $\left[\beta^{\varphi}+\gamma^{\varphi}+\right.$ $\partial \tilde{\alpha}] \in \mathrm{H}^{2,2}(\mathfrak{m}, \mathfrak{h})$ with $\varphi=0$ is the trivial cohomology class.

Proof. The proof of the first claim is as in Lemma 1 and therefore we omit it.

It follows from Lemma 2 that given any $\alpha \in \operatorname{Hom}\left(\Lambda^{2} \vee, V\right)$, there is a unique $\tilde{\alpha} \in$ $C^{2,1}(\mathfrak{m}, \mathfrak{g})=\operatorname{Hom}(\mathrm{V}, \mathfrak{s o}(\mathrm{V}))$ such that $\partial \tilde{\alpha}=\alpha+\tilde{\beta}+\tilde{\gamma}$, for some $\tilde{\beta} \in \operatorname{Hom}(\mathrm{V} \otimes S, S)$ and 
$\tilde{\gamma} \in \operatorname{Hom}\left(\odot^{2} \mathrm{~S}, \mathfrak{s o}(\mathrm{V})\right)$. Therefore any cochain $\alpha+\beta+\gamma \in \mathrm{C}^{2,2}(\mathfrak{m}, \mathfrak{h})$ may be uniquely written as

$$
\alpha+\beta+\gamma=(\alpha+\beta+\gamma-\partial \tilde{\alpha})+\partial \tilde{\alpha}=(\beta-\tilde{\beta})+(\gamma-\tilde{\gamma})+\partial \tilde{\alpha},
$$

where $\beta-\tilde{\beta} \in \operatorname{Hom}(V \otimes S, S)$ and $\gamma-\tilde{\gamma} \in \operatorname{Hom}\left(\odot^{2} S, \mathfrak{s o}(V)\right)$. If $\alpha+\beta+\gamma$ is a cocycle, then so is $(\beta-\tilde{\beta})+(\gamma-\tilde{\gamma})$, so that by Proposition $7, \beta-\tilde{\beta}=\beta^{\varphi}$ and $\gamma-\tilde{\gamma}=\gamma^{\varphi}$, for some $\varphi \in \Lambda^{4} V$ and where $\beta^{\varphi}$ and $\gamma^{\varphi}$ are given by the expressions in Proposition 7. In other words,

$$
\left.\operatorname{ker} \partial\right|_{\mathbf{C}^{2,2}(\mathfrak{m}, \mathfrak{h})} \subset \Lambda^{4} \mathrm{~V} \oplus \partial\left(\mathfrak{s o}(\mathrm{V}) \otimes \mathrm{V}^{*}\right)
$$

where we identified any $\varphi \in \Lambda^{4} V$ with the corresponding cocycle $\beta^{\varphi}+\gamma^{\varphi}$. Now Eq. (7) tells us that

$$
\partial \tilde{\alpha}(s, s)=-\tilde{\alpha}([s, s])
$$

for all $s \in S$ so that an element $\beta^{\varphi}+\gamma^{\varphi}+\partial \tilde{\alpha}$ is in $C^{2,2}(\mathfrak{m}, \mathfrak{h})$ if and only if

$$
\gamma^{\varphi}(s, s)-\tilde{\alpha}([s, s]) \in \mathfrak{h}_{0}
$$

for all $s \in S$. This fact, together with (24), shows that the kernel of $\partial$ restricted to $\mathrm{C}^{2,2}(\mathfrak{m}, \mathfrak{h})$ is given by

$$
\left\{\beta^{\varphi}+\gamma^{\varphi}+\partial \tilde{\alpha} \mid \varphi \in \Lambda^{4} V, \tilde{\alpha}: V \rightarrow \mathfrak{s o}(V) \text { with } \gamma^{\varphi}(s, s)-\tilde{\alpha}([s, s]) \in \mathfrak{h}_{0}\right\}
$$

from which the claim on $\mathrm{H}^{2,2}(\mathfrak{m}, \mathfrak{h})$ follows directly.

The last claim follows from the fact that, if $\varphi=0$, then $\partial \tilde{\alpha}$ satisfies $\tilde{\alpha}([s, s]) \in \mathfrak{h}_{0}$ for all $s \in S$, so that it is in the image of $C^{2,1}(\mathfrak{m}, \mathfrak{h})=\operatorname{Hom}\left(V, \mathfrak{h}_{0}\right)$.

To state our first main result on filtered deformations $F$ of $\mathfrak{h}$ we recall that the Lie brackets of $F$ have components of nonzero filtration degree: the component $\mu$ of degree 2 (see Eq. (5)) and the component $\delta: \wedge^{2} \bigvee \rightarrow \mathfrak{h}_{0}$ of degree 4.

Theorem 9. Let $\mathfrak{h}=\mathfrak{h}_{-2} \oplus \mathfrak{h}_{-1} \oplus \mathfrak{h}_{0}$ be a $\mathbb{Z}$-graded subalgebra of the Poincaré superalgebra $\mathfrak{g}=\mathrm{V} \oplus \mathrm{S} \oplus \mathfrak{s o}(\mathrm{V})$ which differs only in zero degree, i.e. $\mathfrak{h}_{0} \subset \mathfrak{g}_{0}$ and $\mathfrak{m}=\mathfrak{h}_{-2} \oplus \mathfrak{h}_{-1}=\mathrm{V} \oplus \mathrm{S}$. If $\mathrm{F}$ is a filtered deformations of $\mathfrak{h}$ then:

1. $\left.\mu\right|_{\mathfrak{m} \otimes \mathfrak{m}}$ is a cocycle in $\mathrm{C}^{2,2}(\mathfrak{m}, \mathfrak{h})$ and its cohomology class $\left[\left.\mu\right|_{\mathfrak{m} \otimes \mathfrak{m}}\right] \in \mathrm{H}^{2,2}(\mathfrak{m}, \mathfrak{h})$ is $\mathfrak{h}_{0}$-invariant (that is $\left.\mu\right|_{\mathfrak{m} \otimes \mathfrak{m}}$ is $\mathfrak{h}_{0}$-invariant up to exact terms); and

2. If $\mathrm{F}^{\prime}$ is another filtered deformation of $\mathfrak{h}$ such that $\left[\left.\mu^{\prime}\right|_{\mathfrak{m} \otimes \mathfrak{m}}\right]=\left[\left.\mu\right|_{\mathfrak{m} \otimes \mathfrak{m}}\right]$ then $\mathrm{F}^{\prime}$ is isomorphic to $\mathrm{F}$ as a filtered Lie superalgebra.

Proof. By the results of [37], the maximal transitive prolongation of the supertranslation algebra $\mathfrak{m}$ is the $\mathbb{Z}$-graded Lie superalgebra $\mathfrak{g}^{\infty}=\mathfrak{g}_{-2}^{\infty} \oplus \mathfrak{g}_{-1}^{\infty} \oplus \mathfrak{g}_{0}^{\infty}$ where $\mathfrak{g}_{-2}^{\infty} \oplus \mathfrak{g}_{-1}^{\infty}=\mathfrak{m}$ and $\mathfrak{g}_{0}^{\infty}=\mathfrak{s o}(\mathrm{V}) \oplus \mathbb{R E}$, with $E$ the so-called grading element satisfying $\left.\operatorname{ad}(\mathrm{E})\right|_{\mathfrak{g}_{j}^{\infty}}=j \mathrm{Id}$. It is well-known that maximality is equivalent to the fact that the Spencer cohomology group $H^{d, 1}\left(\mathfrak{m}, \mathfrak{g}^{\infty}\right)=0$ for all $d \geqslant 0$ (see e.g. [16]).

Since $\mathfrak{m}=\mathfrak{h}_{-2} \oplus \mathfrak{h}_{-1}$ but $\mathfrak{h}_{0} \subset \mathfrak{s o}(V) \subset \mathfrak{g}_{0}^{\infty}$ this also implies that $\mathrm{H}^{\mathrm{d}, 1}(\mathfrak{m}, \mathfrak{h})=0$ for all $d \geqslant 1$, that is $\mathfrak{h}$ is a full prolongation of degree $k=1$ in the terminology of [16].

The first claim follows directly from Proposition 2.2 of [16]. Let now $F$ and $F^{\prime}$ be two filtered deformations of $\mathfrak{h}$ such that $\left[\left.\mu\right|_{\mathfrak{m} \otimes \mathfrak{m}}\right]=\left[\left.\mu^{\prime}\right|_{\mathfrak{m} \otimes \mathfrak{m}}\right]$. Then $\left.\left(\mu-\mu^{\prime}\right)\right|_{\mathfrak{m} \otimes \mathfrak{m}}$ is a Spencer coboundary and we may first assume without any loss of generality that 
$\left.\mu\right|_{\mathfrak{m} \otimes \mathfrak{m}}=\left.\mu^{\prime}\right|_{\mathfrak{m} \otimes \mathfrak{m}}$ by Proposition 2.3 of [16]. Moreover, since $\mathfrak{h}$ is a full prolongation of degree $k=1$ (and hence, in particular, of degree $k=2$ ), Proposition 2.6 of [16] applies and we may also assume $\mu=\mu^{\prime}$ without any loss of generality. In other words we just showed that $F^{\prime}$ is isomorphic as a filtered Lie superalgebra to another filtered Lie superalgebra $F^{\prime \prime}$ which satisfies $\mu^{\prime \prime}=\mu$.

Now, given any two filtered deformations $F$ and $F^{\prime}$ of $\mathfrak{h}$ with $\mu=\mu^{\prime}$ it is not difficult to see that $\delta-\delta^{\prime}=\left.\left(\delta-\delta^{\prime}\right)\right|_{\mathfrak{m} \otimes \mathfrak{m}}$ is a Spencer cocycle (use e.g. [16, Eq. 2.6]). However $\mathrm{H}^{4,2}(\mathfrak{m}, \mathfrak{h})=\left.\operatorname{Ker} \partial\right|_{C^{4,2}(\mathfrak{m}, \mathfrak{h})}=0$ by Proposition 8 and hence $\delta=\delta^{\prime}$. This proves that any two filtered deformations $F$ and $F^{\prime}$ of $\mathfrak{h}$ with $\left[\left.\mu^{\prime}\right|_{\mathfrak{m} \otimes \mathfrak{m}}\right]=\left[\left.\mu\right|_{\mathfrak{m} \otimes \mathfrak{m}}\right]$ are isomorphic.

In other words, filtered deformations of $\mathfrak{h}$ are completely determined by the $\mathfrak{h}_{0^{-}}$ invariant elements in $\mathrm{H}^{2,2}(\mathfrak{m}, \mathfrak{h})$, a group which we already calculated in Proposition 8. We emphasise that this result in particular says that the components $\rho=\left.\mu\right|_{\mathfrak{h}_{0} \otimes V}: \mathfrak{h}_{0} \otimes$ $\mathrm{V} \rightarrow \mathfrak{h}_{0}$ and $\delta: \Lambda^{2} \mathrm{~V} \rightarrow \mathfrak{h}_{0}$ of non-zero filtration degree are completely determined by the class $\left[\left.\mu\right|_{\mathfrak{m} \otimes \mathfrak{m}}\right] \in \mathrm{H}^{2,2}(\mathfrak{m}, \mathfrak{h})$ (hence by the components $\alpha, \beta$ and $\gamma$ ), up to isomorphisms of filtered Lie superalgebras.

\section{Integrating the Deformations}

In this section, we will determine the $\mathfrak{h}_{0}$-invariant elements in $\mathrm{H}^{2,2}(\mathfrak{m}, \mathfrak{h})$ and, for each of them, construct a filtered deformation. Let us remark that we do not have at our disposal a bracket à la Nijenhuis-Richardson on $\mathrm{H}^{\bullet, \bullet}(\mathfrak{m}, \mathfrak{h})$ that allows one to write down the obstructions to integrating an infinitesimal deformation in terms of classes in $\mathrm{H}^{\bullet, 3}(\mathfrak{m}, \mathfrak{h})$. Therefore our description of filtered Lie superalgebras will be very explicit and rely on a direct check of the Jacobi identities.

4.1. The non-trivial deformations. By the results of Sect. 3, we need only consider deformations corresponding to $\mathfrak{h}_{0}$-invariant cohomology classes in $\mathrm{H}^{2,2}(\mathfrak{m}, \mathfrak{h})$ with $\varphi \neq$ 0 . Indeed if $\varphi=0$ then $\left[\left.\mu\right|_{\mathfrak{m} \otimes \mathfrak{m}}\right]=0$ by Proposition 8 and Theorem 9 , and the associated Lie superalgebras are nothing but the $\mathbb{Z}$-graded subalgebras of the Poincaré superalgebra.

In determining the $\mathfrak{h}_{0}$-invariant classes in $\mathrm{H}^{2,2}(\mathfrak{m}, \mathfrak{h})$, we will also determine the Lie subalgebras $\mathfrak{h}_{0} \subset \mathfrak{s o}(V)$ for which $H^{2,2}(\mathfrak{m}, \mathfrak{h})^{\mathfrak{h}_{0}} \neq 0$ and hence the graded Lie subalgebras $\mathfrak{h}$ of the Poincaré superalgebra admitting nontrivial filtered deformations. We will show that the condition $\mathrm{H}^{2,2}(\mathfrak{m}, \mathfrak{h})^{\mathfrak{h}_{0}} \neq 0$ turns into a system of quadratic equations for $\varphi$ and $\tilde{\alpha}$ which we will be able to solve. In addition we will find that $\mathfrak{h}_{0}=\mathfrak{h}_{\varphi}$, the Lie algebra of the stabiliser in $\operatorname{SO}(V)$ of $\varphi$; that is, $\mathfrak{h}_{\varphi}=\mathfrak{s o}(\mathrm{V}) \cap \mathfrak{s t a b}(\varphi)$, with $\mathfrak{s t a b}(\varphi)$ the Lie algebra of the stabiliser of $\varphi$ in GL(V). We start with a lemma.

Lemma 10. Let $\beta^{\varphi}+\gamma^{\varphi}+\partial \tilde{\alpha}$ be a cocycle in $C^{2,2}(\mathfrak{m}, \mathfrak{h})$ defining a nontrivial, $\mathfrak{h}_{0^{-}}$ invariant cohomology class in $\mathrm{H}^{2,2}(\mathfrak{m}, \mathfrak{h})$. Then $\mathfrak{h}_{0}$ leaves $\varphi$ invariant. In other words, $\mathfrak{h}_{0} \subset \mathfrak{h}_{\varphi}$

Proof. Let $\beta^{\varphi}+\gamma^{\varphi}+\partial \tilde{\alpha}$ be a cocycle in $C^{2,2}(\mathfrak{m}, \mathfrak{h})$ such that its cohomology class in $\mathrm{H}^{2,2}(\mathfrak{m}, \mathfrak{h})$ is non-trivial and $\mathfrak{h}_{0}$-invariant. For our purposes, it is convenient to consider the decomposition of $\mathfrak{s o}(\mathrm{V})$-modules

$$
\mathrm{C}^{2,2}(\mathfrak{m}, \mathfrak{g})=\operatorname{Hom}\left(\wedge^{2} \mathrm{~V}, \mathrm{~V}\right) \oplus \operatorname{Hom}(\mathrm{V} \otimes \mathrm{S}, \mathrm{S}) \oplus \operatorname{Hom}\left(\odot^{2} \mathrm{~S}, \mathfrak{s o}(\mathrm{V})\right)
$$


and the corresponding $\mathfrak{s o}(\mathrm{V})$-equivariant projections (9). We recall that $\tilde{\alpha}: \mathrm{V} \rightarrow \mathfrak{s o}(\mathrm{V})$ is such that $\gamma^{\varphi}+\pi^{\gamma}(\partial \tilde{\alpha}): \odot^{2} S \rightarrow \mathfrak{h}_{0}$. Now $\varphi$ is nonzero, by Proposition 8 , and for any $x \in \mathfrak{h}_{0}$ there is a $\psi \in C^{2,1}(\mathfrak{m}, \mathfrak{h})=\operatorname{Hom}\left(V, \mathfrak{h}_{0}\right)$ such that $x \cdot\left(\beta^{\varphi}+\gamma^{\varphi}+\partial \tilde{\alpha}\right)=\partial \psi$. In other words,

$$
\begin{array}{r}
x \cdot\left(\pi^{\alpha}(\partial \tilde{\alpha})\right)=\pi^{\alpha}(\partial \psi), \\
x \cdot\left(\beta^{\varphi}+\pi^{\beta}(\partial \tilde{\alpha})\right)=\pi^{\beta}(\partial \psi), \\
x \cdot\left(\gamma^{\varphi}+\pi^{\gamma}(\partial \tilde{\alpha})\right)=\pi^{\gamma}(\partial \psi) .
\end{array}
$$

From Eq. (25) and the $\mathfrak{s o}(\mathrm{V})$-equivariance of $\pi^{\alpha}$ and $\partial$, we have

$$
\left(\pi^{\alpha} \circ \partial\right)(\psi)=x \cdot\left(\pi^{\alpha}(\partial \tilde{\alpha})\right)=\left(\pi^{\alpha} \circ \partial\right)(x \cdot \tilde{\alpha})
$$

and then, since $\pi^{\alpha} \circ \partial: \operatorname{Hom}(\mathrm{V}, \mathfrak{s o}(\mathrm{V})) \rightarrow \operatorname{Hom}\left(\wedge^{2} \mathrm{~V}, \mathrm{~V}\right)$ is an isomorphism by Lemma 2 , it follows that $x \cdot \tilde{\alpha}=\psi$. Equation (26) yields now

$$
\begin{aligned}
\pi^{\beta}(\partial \psi) & =x \cdot\left(\beta^{\varphi}+\pi^{\beta}(\partial \tilde{\alpha})\right) \\
& =x \cdot \beta^{\varphi}+x \cdot \pi^{\beta}(\partial \tilde{\alpha}) \\
& =x \cdot \beta^{\varphi}+\pi^{\beta}(\partial(x \cdot \tilde{\alpha}))=x \cdot \beta^{\varphi}+\pi^{\beta}(\partial \psi)
\end{aligned}
$$

so $x \cdot \beta^{\varphi}=0$ and, by a similar argument starting with Eq. (27), $x \cdot \gamma^{\varphi}=0$ too. This shows that $\varphi$ is invariant by $\mathfrak{h}_{0}$ or, in other words, that $\mathfrak{h}_{0} \subset \mathfrak{h}_{\varphi}$.

It follows from this lemma, that if the cocycle $\beta^{\varphi}+\gamma^{\varphi}+\partial \tilde{\alpha}$ defines a nontrivial, $\mathfrak{h}_{0}$-invariant cohomology class in $\mathrm{H}^{2,2}(\mathfrak{m}, \mathfrak{h})$, then in particular the component $\gamma^{\varphi}+$ $\pi^{\gamma}(\partial \tilde{\alpha})$ in $\operatorname{Hom}\left(\odot^{2} S, \mathfrak{h}_{0}\right)$ actually belongs to $\operatorname{Hom}\left(\odot^{2} S, \mathfrak{h}_{\varphi}\right)$ and this has some strong consequences. To exhibit them, we need a technical lemma.

Lemma 11. The cochain $\gamma^{\varphi}+\pi^{\gamma}(\partial \tilde{\alpha})$ takes values in $\mathfrak{h}_{\varphi}$ if and only if $\varphi \in \Lambda^{4} \vee$ and $\tilde{\alpha} \in \operatorname{Hom}(\mathrm{V}, \mathfrak{s o}(\mathrm{V}))$ satisfy the following three systems of quadrics:

$$
\begin{aligned}
\operatorname{skew}_{\lambda_{1}, \ldots, \lambda_{6}}\left(\varphi^{\lambda_{1} \lambda_{2} \lambda_{3} \lambda_{4}} \varphi^{\lambda_{5}\left[\mu_{1} \mu_{2} \mu_{3}\right.} \eta^{\left.\mu_{4}\right] \lambda_{6}}\right) & =0, \\
\varphi_{\rho \mu \nu}{ }^{\left[\mu_{1}\right.} \varphi^{\left.\mu_{2} \mu_{3} \mu_{4}\right] \rho} & =0, \\
\tilde{\alpha}_{\lambda \rho}{ }^{\left[\mu_{1}\right.} \varphi^{\left.\mu_{2} \mu_{3} \mu_{4}\right] \rho} & =0,
\end{aligned}
$$

where in all three formulae we skew-symmetrise in the $\mu_{i}$ and, in addition, in the first formula we skew-symmetrise in the $\lambda_{i}$ as well, but separately.

Proof. The cochain $\gamma^{\varphi}+\pi^{\gamma}(\partial \tilde{\alpha})$ takes values in $\mathfrak{h}_{\varphi}$ if and only if for every $s \in S$, $\gamma(s, s)-\tilde{\alpha}([s, s]) \in \mathfrak{s o}(V)$ leaves $\varphi$ invariant, where $[s, s]$ stands for the Dirac current of s. Relative to an $\eta$-orthonormal basis, $\varphi=\frac{1}{4 !} \varphi^{\mu_{1} \ldots \mu_{4}} \boldsymbol{e}_{\mu_{1}} \wedge \cdots \wedge \boldsymbol{e}_{\mu_{4}}$ and

$$
\gamma(s, s)(\varphi)=\frac{1}{3 !} \varphi^{\mu_{1} \ldots \mu_{4}} \gamma(s, s)^{\nu}{ }_{\mu_{1}} \boldsymbol{e}_{v} \wedge \boldsymbol{e}_{\mu_{2}} \wedge \cdots \wedge \boldsymbol{e}_{\mu_{4}} .
$$

Using Eq. (19), this becomes

$$
\gamma(s, s)(\varphi)=\frac{1}{3} \varphi^{\mu_{1} \ldots \mu_{4}}\left(\bar{s} \Gamma^{\nu} \beta_{\mu_{1}} s\right) \boldsymbol{e}_{v} \wedge \boldsymbol{e}_{\mu_{2}} \wedge \cdots \wedge \boldsymbol{e}_{\mu_{4}},
$$

where, from Proposition 7 ,

$$
\beta_{\rho}=\frac{1}{4 !} \varphi^{\lambda_{1} \ldots \lambda_{4}}\left(\Gamma_{\rho} \Gamma_{\lambda_{1} \ldots \lambda_{4}}-3 \Gamma_{\lambda_{1} \ldots \lambda_{4}} \Gamma_{\rho}\right) .
$$


On the other hand,

$$
-\tilde{\alpha}([s, s])(\varphi)=\frac{1}{3 !} \varphi^{\mu_{1} \ldots \mu_{4}}\left(\bar{s} \Gamma^{\rho} s\right) \tilde{\alpha}_{\rho}^{\nu} \mu_{1} e_{v} \wedge e_{\mu_{2}} \wedge \cdots \wedge e_{\mu_{4}} .
$$

We insert the expression for $\beta_{\mu_{1}}$ into Eq. (29), multiply in $C \ell(V)$ and keep only the terms $\bar{s} \Gamma_{\mu_{1} \ldots \mu_{p}} s$ for $p=1,2,5$ (or, equivalently, 6 ). When the dust clears, we find that $(\gamma(s, s)-\tilde{\alpha}([s, s]))(\varphi)=0$ if and only if

$$
\begin{aligned}
& \frac{1}{6} \varphi^{\lambda_{1} \ldots \lambda_{4}} \varphi^{\lambda_{5}\left[\mu_{1} \ldots \mu_{3}\right.} \eta^{\left.\mu_{4}\right] \lambda_{6}} \bar{s} \Gamma_{\lambda_{1} \ldots \lambda_{6}} s \\
& \quad+8 \varphi_{\rho \mu \nu}{ }^{\left[\mu_{1}\right.} \varphi^{\left.\mu_{2} \ldots \mu_{4}\right] \rho} \rho_{\bar{s}} \Gamma^{\mu \nu} s-\tilde{\alpha}_{\lambda \rho}{ }^{\left[\mu_{1}\right.} \varphi^{\left.\mu_{2} \ldots \mu_{4}\right]} \rho_{\bar{s}} \Gamma^{\lambda} s=0 .
\end{aligned}
$$

By polarisation on $s$, we see that this is a system of quadrics for $\varphi$ and $\tilde{\alpha}$ with linear parametric dependence on $\odot^{2} S$. Since $\odot{ }^{2} S \cong V \oplus \Lambda^{2} V \oplus \Lambda^{5} V$, the components of this system parametrised by $\mathrm{V}, \Lambda^{2} \mathrm{~V}$ and $\Lambda^{5} \mathrm{~V} \cong \Lambda^{6} \mathrm{~V}$ must be satisfied separately. In other words, the terms proportional to $\bar{s} \Gamma^{\lambda} s, \bar{s} \Gamma^{\mu \nu} s$ and $\bar{s} \Gamma_{\lambda_{1} \ldots \lambda_{6}} s$ must vanish separately, and these are precisely the three systems of quadrics in the lemma.

As we shall see, the quadrics (28) have a very natural interpretation. Our first observation is that the first equation in (28) actually implies the second. To see this, we simply contract the first equation with $\eta_{\lambda_{6} \mu_{4}}$ to obtain

$$
\varphi^{\left[\lambda_{1} \lambda_{2} \lambda_{3} \lambda_{4}\right.} \varphi^{\left.\lambda_{5}\right] \mu_{1} \mu_{2} \mu_{3}}=0,
$$

and we now contract again with $\eta_{\lambda_{5} \mu_{3}}$ to obtain the second equation.

Now recall that a non-zero 4-form $\varphi \in \Lambda^{4} \mathrm{~V}$ is said to be decomposable if

$$
\varphi=v_{1} \wedge v_{2} \wedge v_{3} \wedge v_{4}
$$

for some linearly independent $v_{i} \in \mathrm{V}$. If $\varphi=v_{1} \wedge v_{2} \wedge v_{3} \wedge v_{4}$ is decomposable, then the first equation in (28) (and hence also the second) is satisfied identically. To see this, insert $\varphi^{\lambda_{1} \ldots \lambda_{4}}=v_{1}^{\left[\lambda_{1}\right.} v_{2}^{\lambda_{2}} v_{3}^{\lambda_{3}} v_{4}^{\left.\lambda_{4}\right]}$, into the LHS of the first equation in (28) to obtain

$$
\operatorname{skew}_{\mu_{1}, \ldots, \mu_{4}} \operatorname{skew}_{\lambda_{1}, \ldots, \lambda_{6}}\left(v_{1}^{\left[\lambda_{1}\right.} v_{2}^{\lambda_{2}} v_{3}^{\lambda_{3}} v_{4}^{\left.\lambda_{4}\right]} v_{1}^{\left[\lambda_{5}\right.} v_{2}^{\mu_{1}} v_{3}^{\mu_{2}} v_{4}^{\left.\mu_{3}\right]} \eta^{\mu_{4} \lambda_{6}}\right)
$$

where we skew-symmetrise separately in the $\lambda_{i}$ and the $\mu_{i}$. But notice that every term in this expression contains a factor $v_{i}^{\lambda_{j}} v_{i}^{\lambda_{k}}$ for some $i, j, k$, and this vanishes by symmetry since we skew-symmetrise on the $\lambda_{i}$.

Perhaps more remarkable still is that the converse also holds. Indeed, we recognise Eq. (30) as the Plücker relations (see, e.g., [40, Ch. 1])

$$
\iota_{\chi} \iota_{\theta} \iota_{\zeta} \varphi \wedge \varphi=0
$$

for all $\theta, \zeta, \chi \in \mathrm{V}^{*}$, defining the Plücker embedding of the Grassmannian $\operatorname{Gr}(4, \mathrm{~V})$ of 4-planes in $V$ into the projective space $\mathbb{P}\left(\Lambda^{4} V\right)$. Recall that a decomposable 4-form $\varphi=v_{1} \wedge v_{2} \wedge v_{3} \wedge v_{4}$ defines a plane $\Pi \subset \vee$ by the span of the $\left(v_{i}\right)$ and, conversely, any plane determines a decomposable $\varphi$ up to a nonzero real multiple by taking the 4-form constructed out of wedging the elements in any basis. Hence $\varphi$ is decomposable if and only if it obeys Eq. (32). In other words, we have proved that the first two equations in (28) are satisfied if and only if $\varphi$ is decomposable.

Finally, the third quadric in (28) simply says that the image of $\tilde{\alpha}: \mathrm{V} \rightarrow \mathfrak{s o}(\mathrm{V})$ actually lies in $\mathfrak{h}_{\varphi}$.

In summary, we have proved the following 
Proposition 12. The cochain $\gamma^{\varphi}+\pi^{\gamma}(\partial \tilde{\alpha})$ takes values in $\mathfrak{h}_{\varphi}$ if and only if $\varphi \in \Lambda^{4} \mathrm{~V}$ is decomposable and the image $\operatorname{Im}(\tilde{\alpha}) \subset \mathfrak{h}_{\varphi}$.

To proceed further, we need to classify the decomposable $\varphi$ and the corresponding stabilisers $\mathfrak{h}_{\varphi}$. It is only necessary to classify $\varphi$ up to the action of $\operatorname{CSO}(\mathrm{V})=\mathbb{R}^{\times} \times$ $\mathrm{SO}(\mathrm{V})$.

Lemma 13. Let $\varphi$ and $\varphi^{\prime}$ be decomposable 4-forms in the same orbit of $\mathrm{CSO}(\mathrm{V})$ on $\Lambda^{4} \mathrm{~V}$. Then the corresponding filtered deformations are isomorphic.

Proof. The group $\mathrm{G}_{0}^{\infty}=\mathrm{CSpin}(\mathrm{V})$ with Lie algebra $\mathfrak{g}_{0}^{\infty}=\mathfrak{s o}(\mathrm{V}) \oplus \mathbb{R E}$ is a double-cover of $\operatorname{CSO}(\mathrm{V})$ and it naturally acts on $\mathfrak{g}^{\infty}$ by degree-0 Lie superalgebra automorphisms. Note that the action preserves the Poincaré superalgebra $\mathfrak{g}$, which is an ideal of $\mathfrak{g}^{\infty}$. Now, an element $g \in C S p i n(V)$ sends a $\mathbb{Z}$-graded subalgebra $\mathfrak{h}=\mathrm{V} \oplus \mathrm{S} \oplus \mathfrak{h}_{0}$ of $\mathfrak{g}$ into an (isomorphic) $\mathbb{Z}$-graded subalgebra $\mathfrak{h}^{\prime}=\mathfrak{g} \cdot \mathfrak{h}=\mathrm{V} \oplus \mathrm{S} \oplus\left(\mathrm{g} \cdot \mathfrak{h}_{0}\right)$ of $\mathfrak{g}$. In particular, if $\mathrm{F}$ is a filtered deformation of $\mathfrak{h}$ associated with $\varphi$ then $F^{\prime}=g \cdot F$ is also a filtered deformation of $\mathfrak{h}^{\prime}$, which is associated with $\varphi^{\prime}=\mathrm{g} \cdot \varphi$.

Therefore we must classify the orbits of $\operatorname{CSO}(\mathrm{V})$ in the space of decomposable elements of $\Lambda^{4} \mathrm{~V}$. Other than $\varphi=0$, which is its own orbit, any other decomposable $\varphi$ defines a 4-plane and we can study instead the geometric action of $\mathrm{SO}(\mathrm{V})$ on 4-planes. Unlike the general linear group, $\mathrm{SO}(\mathrm{V})$ does not act transitively on the Grassmannian of 4-planes. Indeed, we can distinguish three kinds of planes, depending on the nature of the restriction of the inner product $\eta$ on $V$ to the plane:

1. $\Pi$ is Euclidean: we will say that $\varphi$ is spacelike;

2. $\Pi$ is Lorentzian: we will say that $\varphi$ is timelike;

3. $\Pi$ is degenerate: we will say that $\varphi$ is lightlike.

Since $\mathrm{SO}(\mathrm{V})$ preserves $\eta$, it preserves the type of plane and acts transitively on each type. In terms of the 4-forms, one can show that, in addition to the trivial orbit $\varphi=0$, there are precisely three orbits of $\mathrm{CSO}(\mathrm{V})$ on the space of decomposable elements in $\Lambda^{4} \mathrm{~V}$.

Many of the results we prove from here on depend on a case-by-case analysis of these three orbits. We find that the first two orbits can be treated simultaneously, since they share the property that the restriction of $\eta$ to $\Pi$ is nondegenerate. In this case, we can decompose $\mathrm{V}=\Pi \oplus \Pi^{\perp}$ into an orthogonal direct sum and hence $\mathfrak{h}_{\varphi}=\mathfrak{s o}(\Pi) \oplus \mathfrak{s o}\left(\Pi^{\perp}\right) \subset$ $\mathfrak{s o}(\mathrm{V})$.

In contrast, if $\Pi$ is degenerate, we can always choose an $\eta$-Witt basis for $V$ such that $V=\mathbb{R}\left\langle\mathbf{e}_{+}, \mathbf{e}_{-}\right\rangle \oplus W$ and such that $\varphi=\mathbf{e}_{+} \wedge f$ for $f \in \Lambda^{3} W$ a decomposable 3-form. Such $f$ defines a 3-plane $\pi \subset W$ and induces an orthogonal decomposition $W=\pi \oplus \pi^{\perp}$. Our original plane is $\Pi=\mathbb{R}\left\langle\mathbf{e}_{+}\right\rangle \oplus \pi$ and the stabiliser Lie algebra is now

$$
\mathfrak{h}_{\varphi}=\left(\mathfrak{s o}(\pi) \oplus \mathfrak{s o}\left(\pi^{\perp}\right)\right) \ltimes\left(\mathbf{e}_{+} \wedge\left(\pi \oplus \pi^{\perp}\right)\right) \subset \mathfrak{s o}(\mathrm{V})
$$

where

$$
\mathbf{e}_{+} \wedge\left(\pi \oplus \pi^{\perp}\right)=\left(\mathbf{e}_{+} \wedge \pi\right) \oplus\left(\mathbf{e}_{+} \wedge \pi^{\perp}\right),
$$

is the abelian Lie subalgebra of $\mathfrak{s o}(\mathrm{V})$ consisting of null rotations fixing $\mathbf{e}_{+}$. We remark that whether or not $\Pi$ is degenerate, $\operatorname{dim} \mathfrak{h}_{\varphi}=27$ and in fact the degenerate $\mathfrak{h}_{\varphi}$ is a contraction of the nondegenerate $\mathfrak{h}_{\varphi}$.

We are now ready to prove the following proposition, which recapitulates the results of this section. 
Proposition 14. Let $\mathfrak{h}=\mathfrak{h}_{-2} \oplus \mathfrak{h}_{-1} \oplus \mathfrak{h}_{0}$ be a $\mathbb{Z}$-graded subalgebra of the Poincaré superalgebra $\mathfrak{g}$ which differs only in zero degree; that is, $\mathfrak{h}_{0} \subset \mathfrak{g}_{0}$ and $\mathfrak{m}=\mathfrak{h}_{-2} \oplus \mathfrak{h}-1=$ $\mathrm{V} \oplus \mathrm{S}$. In addition, let $\beta^{\varphi}+\gamma^{\varphi}+\partial \tilde{\alpha}$ be a cocycle in $\mathrm{C}^{2,2}(\mathfrak{m}, \mathfrak{h})$ defining a nontrivial, $\mathfrak{h}_{0}$-invariant cohomology class in $\mathrm{H}^{2,2}(\mathfrak{m}, \mathfrak{h})$. Then,

1. $\varphi \in \Lambda^{4} \mathrm{~V}$ is nonzero and decomposable,

2. The images $\operatorname{Im}\left(\gamma^{\varphi}\right)=\mathfrak{h}_{\varphi}$ and $\operatorname{Im}(\tilde{\alpha}) \subset \mathfrak{h}_{\varphi}$, and

3. $\mathfrak{h}_{0}=\mathfrak{h}_{\varphi}$.

Proof. The first part follows from Lemma 10 and Proposition 12. Further, from Lemma 10 we have that for all $s \in S$,

$$
\gamma^{\varphi}(s, s)-\tilde{\alpha}([s, s]) \in \mathfrak{h}_{\varphi}
$$

but from Proposition 12 we also know that $\tilde{\alpha}([s, s]) \in \mathfrak{h}_{\varphi}$, hence $\operatorname{Im}\left(\gamma^{\varphi}\right) \subset \mathfrak{h}_{\varphi}$ as well.

To prove the second part, we need to show that $\operatorname{Im}\left(\gamma^{\varphi}\right)=\mathfrak{h}_{\varphi}$. We break this up into two cases, depending on whether or not the plane $\Pi$ corresponding to $\varphi$ is degenerate.

$\Pi$ is nondegenerate. From Proposition 7, we have that

$$
\eta\left(w, \gamma^{\varphi}(s, s) v\right)=2\left\langle s, w \cdot \beta_{v}^{\varphi} \cdot s\right\rangle
$$

Writing $v=v_{\top}+v_{\perp}$ and $w=w_{\top}+w_{\perp}$, and using that $\beta_{\nu}^{\varphi}=4 v_{\top} \cdot \varphi-2 v_{\perp} \cdot \varphi$ we arrive after some calculation at

$$
\eta\left(w, \gamma^{\varphi}(s, s) v\right)=8\left\langle s, \iota_{w_{\top}} \iota_{v_{\top}} \varphi \cdot s\right\rangle-4\left\langle s, w_{\perp} \wedge v_{\perp} \wedge \varphi \cdot s\right\rangle
$$

In other words, $\gamma^{\varphi}$ defines a map

$$
\odot^{2} \mathrm{~S} \rightarrow \mathfrak{s o}(\Pi) \oplus \mathfrak{s o}\left(\Pi^{\perp}\right)
$$

which we claim is surjective. Indeed, the only way that the first component of this map fails to be surjective is if there exists a nonzero $\zeta \in \Lambda^{2} \Pi$ such that

$$
\left\langle s, \iota_{\zeta} \varphi \cdot s\right\rangle=0 \quad \forall s \in S
$$

From Eq. (42), this is true if and only if $\iota_{\zeta} \varphi=0$, but this implies that $\zeta=0$. Similarly, the second component of the map would fail to be surjective if there exists a nonzero $\theta \in \Lambda^{2} \Pi^{\perp}$ such that

$$
\langle s, \theta \wedge \varphi \cdot s\rangle=0 \quad \forall s \in S
$$

In turn, this is equivalent to

$$
\langle s, \star(\theta \wedge \varphi) \cdot s\rangle=0 \quad \forall s \in S,
$$

which, by (43), implies that $\star(\theta \wedge \varphi)=0$ or, equivalently, $\theta \wedge \varphi=0$; but no nonzero $\theta \in \Lambda^{2} \Pi^{\perp}$ has vanishing wedge product with $\varphi$. Therefore $\theta=0$.

$\Pi$ is degenerate. In this case we can write $w=w_{+}+w_{-}+w_{\top}+w_{\perp}$ and now

$$
\beta_{v}^{\varphi}=4 v_{\top} \cdot \varphi-2 v_{\perp} \cdot \varphi-2 v_{-} \cdot \varphi-6 \theta\left(v_{-}\right) f
$$


where $\varphi=e_{+} \wedge f$ and $\theta\left(\nu_{-}\right)=\eta\left(\nu, e_{+}\right)$. After a short calculation, we arrive at

$$
\begin{aligned}
\eta\left(w, \gamma^{\varphi}(s, s) v\right)= & 2\left\langle s, w \cdot \beta_{v}^{\varphi} \cdot s\right\rangle \\
= & 8 \theta\left(w_{-}\right)\left\langle s, \iota_{v_{\top}} f \cdot s\right\rangle-8 \theta\left(v_{-}\right)\left\langle s, \iota_{w_{\top}} f \cdot s\right\rangle \\
& -4\left\langle s, w_{\perp} \wedge v_{\perp} \wedge \varphi \cdot s\right\rangle+8\left\langle s, \iota_{w_{\top}} \iota_{v_{\top}} \varphi \cdot s\right\rangle \\
& -4\left\langle s, w_{-} \wedge v_{\perp} \wedge \varphi \cdot s\right\rangle+4\left\langle s, v_{-} \wedge w_{\perp} \wedge \varphi \cdot s\right\rangle .
\end{aligned}
$$

The first two terms factor through the component $\odot^{2} S \rightarrow\left(\boldsymbol{e}_{+} \wedge \pi\right)$ of $\gamma^{\varphi}$, whereas the second pair of terms factor through the component $\odot^{2} S \rightarrow\left(\mathbf{e}_{+} \wedge \pi^{\perp}\right)$. The last two terms factor through the components $\odot^{2} S \rightarrow \mathfrak{s o}(\pi)$ and $\odot^{2} S \rightarrow \mathfrak{s o}\left(\pi^{\perp}\right)$ of $\gamma^{\varphi}$, respectively. Similar arguments to the ones in the nondegenerate case show that these maps are surjective.

Finally, we show that $\mathfrak{h}_{0}=\mathfrak{h}_{\varphi}$. From Lemma 10 we know that $\mathfrak{h}_{0} \subset \mathfrak{h}_{\varphi}$, so all we need to do is to establish the reverse inclusion: $\mathfrak{h}_{0} \supset \mathfrak{h}_{\varphi}$. This will follow from

$$
\mathfrak{h}_{0} \supset \operatorname{Im}\left(\gamma^{\varphi}+\pi^{\gamma}(\partial \tilde{\alpha})\right)=\operatorname{Im}\left(\gamma^{\varphi}\right)+\operatorname{Im}\left(\pi^{\gamma}(\partial \tilde{\alpha})\right)=\mathfrak{h}_{\varphi},
$$

where the first equality is a consequence of the fact, to be shown, that we may actually think of $\gamma^{\varphi}+\pi^{\gamma}(\partial \tilde{\alpha})$ as

$$
\gamma^{\varphi} \oplus \pi^{\gamma}(\partial \tilde{\alpha}):\left(\Lambda^{2} V \oplus \Lambda^{5} \mathrm{~V}\right) \oplus \mathrm{V} \rightarrow \mathfrak{h}_{\varphi}
$$

where we identify $\odot{ }^{2} S$ with the direct sum $V \oplus \Lambda^{2} V \oplus \Lambda^{5} V$ of subspaces $\Lambda^{p} V \subset \odot^{2} S$, for $p=1,2,5$, defined by Eq. (44).

It follows from the very definition of $\pi^{\gamma}(\partial \tilde{\alpha})$ that it is given by a map $V \rightarrow \mathfrak{h}_{\varphi}$ : in fact, the map is precisely $-\tilde{\alpha}$. We now use equations (45) to calculate

$$
\eta\left(w, \gamma^{\varphi}(s, s) v\right)=4\langle s, v \wedge w \wedge \varphi \cdot s\rangle-8\left\langle s, \iota_{v} \iota_{w} \varphi \cdot s\right\rangle,
$$

which shows that $\gamma^{\varphi}: \Lambda^{2} \vee \oplus \Lambda^{5} \vee \rightarrow \mathfrak{h}_{\varphi}$, where we have used that $\Lambda^{6} \vee \cong \Lambda^{5} \vee$

4.2. First-order integrability of the deformation. From Propositions 8, 14, Theorem 9, Lemma 13 we know that there are (at most) three isomorphism classes of non-trivial filtered deformations of subalgebras $\mathfrak{h}=\mathrm{V} \oplus \mathrm{S} \oplus \mathfrak{h}_{0}$ of the Poincaré superalgebra, each one determined by a nonzero decomposable $\varphi \in \Lambda^{4} \mathrm{~V}$ which can be either spacelike, timelike or lightlike. Moreover $\mathfrak{h}_{0}=\mathfrak{h}_{\varphi}=\mathfrak{s o}(V) \cap \mathfrak{s t a b}(\varphi)$ and

$$
H^{2,2}(\mathfrak{m}, \mathfrak{h})^{\mathfrak{h}_{0}}=\frac{\left\{\beta^{\varphi}+\gamma^{\varphi}+\partial \tilde{\alpha} \mid \tilde{\alpha}: V \rightarrow \mathfrak{h}_{0}\right\}}{\left\{\partial \tilde{\alpha} \mid \tilde{\alpha}: V \rightarrow \mathfrak{h}_{0}\right\}} \cong\left\{\beta^{\varphi}+\gamma^{\varphi} \mid \varphi \in\left(\Lambda^{4} \mathrm{~V}\right)^{\mathfrak{h}_{0}}\right\} .
$$

In other words the action of $\mu$ on $V \otimes S$ and $\odot^{2} S$ is given by $\beta^{\varphi}$ and $\gamma^{\varphi}$ (recall that $\gamma^{\varphi}: \odot{ }^{2} \mathrm{~S} \rightarrow \mathfrak{s o}(\mathrm{V})$ already takes values in $\mathfrak{h}_{0}$ when $\varphi$ is decomposable) and one can always assume $\alpha=\left.\mu\right|_{\Lambda^{2} \bigvee}=0$ without loss of generality.

Let us now introduce a formal parameter $t$ to keep track of the order of the deformation. The original graded Lie superalgebra structure has order $t^{0}$ and the infinitesimal deformation has order $t$. We will now show that, to first order in $t$, the filtered Lie superalgebra structure on $\mathfrak{h}$ is given by

$$
\begin{aligned}
{\left[v_{1}, v_{2}\right] } & =0 \\
{[v, s] } & =\mathrm{t} \beta_{\nu}^{\varphi}(s)=\mathrm{t}(\nu \cdot \varphi-3 \varphi \cdot v) \cdot \mathrm{s} \\
{\left[s_{1}, s_{2}\right] } & =\left[s_{1}, s_{2}\right]_{0}+\mathrm{t} \gamma^{\varphi}\left(s_{1}, s_{2}\right),
\end{aligned}
$$


where $\left[s_{1}, s_{2}\right]_{0}$ denotes the original Lie bracket defined by Eq. (4) and the brackets involving $\mathfrak{h}_{0}$ are unchanged. In particular we set $\rho=\left.\mu\right|_{\mathfrak{h}_{0} \otimes V}: \mathfrak{h}_{0} \otimes \mathrm{V} \rightarrow \mathfrak{h}_{0}$ to be zero.

We now check that all the Jacobi identities are satisfied to first order in $t$. For example, the identity

$$
[\lambda,[v, s]]=[[\lambda, v], s]+[v,[\lambda, s]]
$$

for $\lambda \in \mathfrak{h}_{0}, v \in \mathrm{V}$ and $s \in S$, is equivalent to the $\lambda$-equivariance of the $[v, s]$ bracket and it is indeed satisfied: this bracket is not zero but depends on $\varphi$ which is left invariant by $\lambda$. To go through all the identities systematically, we use the notation $[i j k]$ for $i, j, k=0,1,2$ to denote the identity involving $X \in \mathfrak{h}_{-i}, Y \in \mathfrak{h}_{-j}$ and $Z \in \mathfrak{h}_{-k}$ :

- The [000] Jacobi identity is satisfied by virtue of $\mathfrak{h}_{0}=\mathfrak{h}_{\varphi}$ being a Lie algebra;

- The [001] and [002] Jacobi identities are satisfied because $S$ and $V$ are $\mathfrak{h}_{\varphi}$-modules (by restriction);

- The [011], [012] and [022] Jacobi identities are satisfied because the [SS], [SV] Lie brackets are $\mathfrak{h}_{\varphi}$-equivariant;

- The [112] and [111] Jacobi identities are satisfied by virtue of the first and second cocycle conditions (10) and (11), respectively;

- The [122] and [222] Jacobi identities are trivially satisfied to first order in $t$.

4.3. All-orders integrability of the deformation. Although the [122] Jacobi identity is satisfied to first order in $t$, it experiences an obstruction at second order. Indeed, for all $s \in \mathrm{S}$ and $v_{1}, v_{2} \in \mathrm{V}$, the [122] Jacobi identity is

$$
\left[\left[v_{1}, v_{2}\right], s\right] \stackrel{?}{=}\left[v_{1},\left[v_{2}, s\right]\right]-\left[v_{2},\left[v_{1}, s\right]\right]=\mathrm{t}^{2}\left[\beta_{v_{1}}^{\varphi}, \beta_{v_{2}}^{\varphi}\right](s)
$$

One can check that $\beta_{\nu_{1}}^{\varphi} \beta_{\nu_{2}}^{\varphi} \neq \beta_{\nu_{2}}^{\varphi} \beta_{\nu_{1}}^{\varphi}$ in general, so that we need to cancel this by modifying the $\left[v_{1}, v_{2}\right]$ bracket. The following lemma suggests how to do this.

Lemma 15. For all $v, w \in V$, the commutator $\left[\beta_{v}^{\varphi}, \beta_{w}^{\varphi}\right]$ lies in the image of $\mathfrak{h}_{\varphi}$ in $\operatorname{End}(S)$.

Proof. There are three cases to consider, depending on whether $\varphi$ is timelike, spacelike or lightlike. In all cases, $\varphi^{2}=\varphi \cdot \varphi \in \operatorname{End}(S)$ is a scalar multiple of the identity: positive if $\varphi$ is spacelike, negative if $\varphi$ is timelike and zero if $\varphi$ is lightlike. In the first two cases, the 4-plane $\Pi \subset \mathrm{V}$ determined by $\varphi$ is nondegenerate and we may decompose $V=\Pi \oplus \Pi^{\perp}$. We tackle these cases first and then finally the case where $\Pi$ is degenerate.

$\Pi$ is nondegenerate. In this case $\varphi^{2}$ is a nonzero multiple of the identity. If $\nu \in \Pi$, then $v \cdot \varphi=-\varphi \cdot v$ and $\beta_{v}^{\varphi}=4 v \cdot \varphi$, whereas if $v \in \Pi^{\perp}$, then $v \cdot \varphi=\varphi \cdot v$ and $\beta_{v}^{\varphi}=-2 v \cdot \varphi$. In general, we can decompose any $v \in \mathrm{V}$ as $v=v_{\top}+v_{\perp}$ with $v_{\top} \in \Pi$ and $v_{\perp} \in \Pi^{\perp}$, and $\beta_{\nu}^{\varphi}=4 v_{\top} \cdot \varphi-2 v_{\perp} \cdot \varphi$. The commutator is given by

$$
\begin{aligned}
{\left[\beta_{v}^{\varphi}, \beta_{w}^{\varphi}\right]=} & {\left[4 v_{\top} \cdot \varphi-2 v_{\perp} \cdot \varphi, 4 w_{\top} \cdot \varphi-2 w_{\perp} \cdot \varphi\right] } \\
= & 16\left[v_{\top} \cdot \varphi, w_{\top} \cdot \varphi\right]+4\left[v_{\perp} \cdot \varphi, w_{\perp} \cdot \varphi\right] \\
& -8\left[v_{\top} \cdot \varphi, w_{\perp} \cdot \varphi\right]-8\left[v_{\perp} \cdot \varphi, w_{\top} \cdot \varphi\right] \\
= & -16\left[v_{\top}, w_{\top}\right] \cdot \varphi^{2}+4\left[v_{\perp}, w_{\perp}\right] \cdot \varphi^{2}+16 \eta\left(v_{\top}, w_{\perp}\right) \varphi^{2}-16 \eta\left(v_{\perp}, w_{\top}\right) \varphi^{2} \\
= & -16\left[v_{\top}, w_{\top}\right] \cdot \varphi^{2}+4\left[v_{\perp}, w_{\perp}\right] \cdot \varphi^{2} .
\end{aligned}
$$


Notice that $\varphi^{2}$ is a (nonzero) scalar endomorphism, so that $\left[\beta_{v}^{\varphi}, \beta_{w}^{\varphi}\right]$ lies in the image of $\mathfrak{s o}(\mathrm{V})$ in $\mathrm{C} \ell(\mathrm{V})$. Moreover, both $\left[v_{\top}, w_{\top}\right]$ and $\left[v_{\perp}, w_{\perp}\right]$ commute with $\varphi$ in $\mathrm{C} \ell(\mathrm{V})$, whence $\left[\beta_{v}^{\varphi}, \beta_{w}^{\varphi}\right]$ also lies in the image of $\mathfrak{s t a b}(\varphi)$.

$\Pi$ is degenerate. In this case, $\mathrm{V}=\mathbb{R}\left\langle\mathbf{e}_{+}, \mathbf{e}_{-}\right\rangle \oplus \pi \oplus \pi^{\perp}$ and thus any $v \in \mathrm{V}$ admits a unique decomposition $v=v_{+}+v_{-}+v_{\top}+v_{\perp}$, where $v_{ \pm} \in \mathbb{R} \mathbf{e}_{ \pm}, v_{\top} \in \pi$ and $v_{\perp} \in \pi^{\perp}$. Now we still have that $\nu_{\top} \cdot \varphi=-\varphi \cdot \nu_{\top}, \nu_{\perp} \cdot \varphi=\varphi \cdot v_{\perp}$, but also $\nu_{+} \cdot \varphi=\varphi \cdot v_{+}=0$ and $v_{-} \cdot \varphi-\varphi \cdot v_{-}=-2 \eta\left(v_{-}, e_{+}\right)$f. Let us abbreviate $\eta\left(\nu, e_{+}\right)$by $\theta\left(v_{-}\right)$, so that $\varphi \cdot v_{-} \cdot \varphi=-2 \theta\left(v_{-}\right) \Gamma_{+} \cdot f^{2}$ and notice that $\beta_{\nu}^{\varphi}=4 v_{\top} \cdot \varphi-2 v_{\perp} \cdot \varphi-2 v_{-} \cdot \varphi-6 \theta\left(v_{-}\right) f$. We now calculate (omitting the $\cdot$ notation):

$$
\begin{aligned}
\frac{1}{4}\left[\beta_{v}^{\varphi}, \beta_{w}^{\varphi}\right]= & {\left[2 v_{\top} \varphi-v_{\perp} \varphi-v_{-} \varphi-3 \theta\left(v_{-}\right) f, 2 w_{\top} \varphi-w_{\perp} \varphi-w_{-} \varphi-3 \theta\left(w_{-}\right) f\right] } \\
= & 4\left[v_{\top} \varphi, w_{\top} \varphi\right]-2\left[v_{\top} \varphi, w_{\perp} \varphi\right]-2\left[v_{\top} \varphi, w_{-} \varphi\right]-6 \theta\left(w_{-}\right)\left[v_{\top} \varphi, f\right] \\
& -2\left[v_{\perp} \varphi, w_{\top} \varphi\right]+\left[v_{\perp} \varphi, w_{\perp} \varphi\right]+\left[v_{\perp} \varphi, w_{-} \varphi\right]+3 \theta\left(w_{-}\right)\left[v_{\perp} \varphi, f\right] \\
& -2\left[v_{-} \varphi, w_{\top} \varphi\right]+\left[v_{-} \varphi, w_{\perp} \varphi\right]+\left[v_{-} \varphi, w_{-} \varphi\right]+3 \theta\left(w_{-}\right)\left[v_{-} \varphi, f\right] \\
& -6 \theta\left(v_{-}\right)\left[f, w_{\top} \varphi\right]+3 \theta\left(v_{-}\right)\left[f, w_{\perp} \varphi\right]+3 \theta\left(v_{-}\right)\left[f, w_{-} \varphi\right] .
\end{aligned}
$$

Many of these terms vanish, namely:

$$
\left[v_{\top} \varphi, w_{\top} \varphi\right]=\left[v_{\perp} \varphi, w_{\perp} \varphi\right]=\left[v_{\top} \varphi, w_{\perp} \varphi\right]=0, \quad\left[v_{\perp} \varphi, f\right]=\left[v_{-} \varphi, f\right]=0,
$$

whereas we have that

$$
\begin{aligned}
{\left[v_{\top} \varphi, w_{-} \varphi\right] } & =-2 \theta\left(w_{-}\right) v_{\top} \Gamma_{+} f^{2} \\
{\left[v_{\top} \varphi, f\right] } & =2 v_{\top} \Gamma_{+} f^{2} \\
{\left[v_{\perp} \varphi, w_{-} \varphi\right] } & =-2 \theta\left(w_{-}\right) v_{\perp} \Gamma_{+} f^{2} \\
{\left[v_{-} \varphi, w_{-} \varphi\right] } & =2\left(\theta\left(v_{-}\right) w_{-}-\theta\left(w_{-}\right) v_{-}\right) \Gamma_{+} f^{2} .
\end{aligned}
$$

Putting it all together we arrive at

$$
\begin{aligned}
\frac{1}{4}\left[\beta_{v}^{\varphi}, \beta_{w}^{\varphi}\right]= & \theta\left(v_{-}\right)\left(8 w_{\top}+2 w_{\perp}\right) \Gamma_{+} f^{2}-\theta\left(w_{-}\right)\left(8 v_{\top}+2 v_{\perp}\right) \Gamma_{+} f^{2} \\
& +\left(\theta\left(v_{-}\right) w_{-}-\theta\left(w_{-}\right) v_{-}\right) \Gamma_{+} f^{2} \\
= & \theta\left(v_{-}\right)\left(8 w_{\top}+2 w_{\perp}\right) \Gamma_{+} f^{2}-\theta\left(w_{-}\right)\left(8 v_{\top}+2 v_{\perp}\right) \Gamma_{+} f^{2},
\end{aligned}
$$

where the last equality follows from the fact that both $v_{-}$and $w_{-}$are proportional to the vector $e_{-}$. Since $f^{2}$ is a nonzero scalar multiple of the identity, we see that both terms in the RHS are in the image of $\mathfrak{s o}(\mathrm{V})$ in $\mathrm{C} \ell(\mathrm{V})$ and clearly also in the image of $\mathfrak{s t a b}(\varphi)$, due to the presence of the $\Gamma_{+}$.

Let us then define $\delta: \wedge^{2} \vee \rightarrow \mathfrak{h}_{\varphi}$ by

$$
\left[\delta\left(v_{1}, v_{2}\right), s\right]=\left[\beta_{v_{1}}^{\varphi}, \beta_{v_{2}}^{\varphi}\right](s)
$$

for all $s \in \mathrm{S}$ and modify the [VV] Lie bracket as

$$
\left[v_{1}, v_{2}\right]=t^{2} \delta\left(v_{1}, v_{2}\right)
$$

so that the [122] Jacobi identity is now satisfied to order $t^{2}$. More is true, however, and all Jacobi identities are now satisfied for all $t$.

We may summarise our results as follows: 
Theorem 16. Let $\varphi \in \Lambda^{4} \vee$ be decomposable and let $\mathrm{F}=\mathrm{F}_{\overline{0}} \oplus \mathrm{F}_{\overline{1}}$ be a $\mathbb{Z}_{2}$-graded vector space with $\mathrm{F}_{\overline{0}}=\mathrm{V} \oplus \mathfrak{h}_{\varphi}$ and $\mathrm{F}_{\overline{1}}=\mathrm{S}$, where $\mathfrak{h}_{\varphi}=\mathfrak{s o}(\mathrm{V}) \cap \mathfrak{s t a b}(\varphi)$. The Lie brackets

$$
\left[v_{1}, v_{2}\right]=\mathrm{t}^{2} \delta\left(v_{1}, v_{2}\right), \quad[v, s]=\mathrm{t} \beta_{v}^{\varphi}(\mathrm{s}), \quad\left[\mathrm{s}_{1}, \mathrm{~s}_{2}\right]=\left[\mathrm{s}_{1}, \mathrm{~s}_{2}\right]_{0}+\mathrm{t} \gamma^{\varphi}\left(\mathrm{s}_{1}, \mathrm{~s}_{2}\right),
$$

with $\beta^{\varphi}, \gamma^{\varphi}$ and $\delta$ given by Eqs. (18) and (34), together with the Dirac current $\left[\mathrm{s}_{1}, \mathrm{~s}_{2}\right]_{0}$ as in (4) and the adjoint action of $\mathfrak{h}_{\varphi}$ on itself and its actions on $\mathrm{S}$ and $\mathrm{V}$ given by restricting the spinor and vector representations of $\mathfrak{s o}(\mathrm{V})$, respectively, define on $\mathrm{F} a$ structure of a Lie superalgebra for all $\mathrm{t}$.

Moreover any filtered deformation of a $\mathbb{Z}$-graded subalgebra of the Poincaré superalgebra which differs only in zero degree is of this form.

Proof. Two Jacobi components remain to be checked: the [112] component, which is equivalent to

$$
\delta\left(\nu,[s, s]_{0}\right) \stackrel{?}{=} 2 \gamma^{\varphi}\left(\beta_{v}^{\varphi}(\mathrm{s}), \mathrm{s}\right) \quad \forall \mathrm{s} \in \mathrm{S}, \nu \in \mathrm{V},
$$

and the [222] component, which is equivalent to

$$
[\delta(v, w), u]+[\delta(w, u), v]+[\delta(u, v), w] \stackrel{?}{=} 0 \quad \forall u, v, w \in \mathrm{V} .
$$

As in the proof of Lemma 15, we prove the identities (35) and (36) by calculating in $\mathrm{C} \ell(\mathrm{V})$ and breaking the calculation into two cases, according to whether or not the plane associated with $\varphi$ is degenerate. In proving identity (35) we will use the abbreviation $z:=[s, s]_{0}$.

$\Pi$ is nondegenerate. In the proof of Lemma 15 we derived the expression

$$
\delta(v, w)=-16\left[v_{\top}, w_{\top}\right] \cdot \varphi^{2}+4\left[v_{\perp}, w_{\perp}\right] \cdot \varphi^{2},
$$

for any $v, w \in V$. Let us calculate, for $z=[s, s]_{0}$,

$$
\eta([\delta(v, z), w], u)=-16 \eta\left(\left[\left[v_{\top}, z_{\top}\right], w_{\top}\right], u_{\top}\right)+4 \eta\left(\left[\left[v_{\perp}, z_{\perp}\right], w_{\perp}\right], u_{\perp}\right) .
$$

Using that

$$
[[v, z], w]=4 \eta(v, w) z-4 \eta(z, w) v
$$

we can write

$$
\begin{aligned}
\eta([\delta(v, z), w], u)= & -64 \eta\left(v_{\top}, w_{\top}\right) \eta\left(z_{\top}, u_{\top}\right)+64 \eta\left(z_{\top}, w_{\top}\right) \eta\left(v_{\top}, u_{\top}\right) \\
& +16 \eta\left(v_{\perp}, w_{\perp}\right) \eta\left(z_{\perp}, u_{\perp}\right)-16 \eta\left(z_{\perp}, w_{\perp}\right) \eta\left(v_{\perp}, u_{\perp}\right) .
\end{aligned}
$$

On the other hand,

$$
\eta\left(2\left[\gamma^{\varphi}\left(\beta_{v}^{\varphi}(s), s\right), w\right], u\right)=-2\left\langle u \cdot \beta_{w}^{\varphi} \cdot \beta_{v}^{\varphi} \cdot s, s\right\rangle-2\left\langle\sigma\left(\beta_{v}^{\varphi}\right) \cdot u \cdot \beta_{w}^{\varphi} \cdot s, s\right\rangle,
$$

where $\sigma$ is the anti-involution in $C \ell(V)$ defined by the symplectic structure. One calculates in $\mathrm{C} \ell(\mathrm{V})$ to find

$$
\sigma\left(\beta_{v}^{\varphi}\right) \cdot u \cdot \beta_{w}^{\varphi}=4\left(2 v_{\top}+v_{\perp}\right)\left(u_{\top}-u_{\perp}\right)\left(2 w_{\top}+w_{\perp}\right),
$$

and

$$
u \cdot \beta_{w}^{\varphi} \cdot \beta_{v}^{\varphi}=4\left(u_{\top}+u_{\perp}\right)\left(w_{\perp} v_{\perp}+2 w_{\perp} v_{\top}-2 w_{\top} v_{\perp}-4 w_{\top} v_{\top}\right)
$$


and hence

$$
\eta\left(2\left[\gamma^{\varphi}\left(\beta_{v}^{\varphi}(s), s\right), w\right], u\right)=-16\left\langle s,\left[\left[u_{\top}, w_{\top}\right], v_{\top}\right] \cdot s\right\rangle+4\left\langle s,\left[\left[u_{\perp}, w_{\perp}\right], v_{\perp}\right] \cdot s\right\rangle .
$$

We use Eq. (38) again to arrive at

$$
\begin{aligned}
\eta\left(2\left[\gamma^{\varphi}\left(\beta_{v}^{\varphi}(s), s\right), w\right], u\right)= & -64 \eta\left(u_{\top}, v_{\top}\right)\left\langle s, w_{\top} \cdot s\right\rangle+64 \eta\left(w_{\top}, v_{\top}\right)\left\langle s, u_{\top} \cdot s\right\rangle \\
& +16 \eta\left(u_{\perp}, v_{\perp}\right)\left\langle s, w_{\perp} \cdot s\right\rangle-16 \eta\left(w_{\perp}, v_{\perp}\right)\left\langle s, u_{\perp} \cdot s\right\rangle,
\end{aligned}
$$

which agrees with $\eta([\delta(\nu, z), w], u)$ after using the definition of the Dirac current.

To prove the identity (36), we again depart from the expression (37) for $\delta(v, w)$, so that in $\mathrm{C} \ell(\mathrm{V})$,

$$
\begin{aligned}
{[\delta(v, w), u] } & =\left[-16\left[v_{\top}, w_{\top}\right] \cdot \varphi^{2}+4\left[v_{\perp}, w_{\perp}\right] \cdot \varphi^{2}, u_{\top}+u_{\perp}\right] \\
& =-16\left[\left[v_{\top}, w_{\top}\right], u_{\top}\right] \cdot \varphi^{2}+4\left[\left[v_{\perp}, w_{\perp}\right], u_{\perp}\right] \cdot \varphi^{2},
\end{aligned}
$$

using that $\varphi^{2}$ is central and the fact that $\left[v_{\top}, w_{\top}\right] \in \mathfrak{s o}(\Pi)\left(\operatorname{resp} .\left[\nu_{\perp}, w_{\perp}\right] \in \mathfrak{s o}\left(\Pi^{\perp}\right)\right)$ acts trivially on $\Pi^{\perp}$ (resp. $\Pi$ ). It is clear that the [222] Jacobi identity follows in this case from the Jacobi identity of the commutator in the associative algebra $\mathrm{C} \ell(\mathrm{V})$.

$\Pi$ is degenerate. This case is computationally more involved, but it is again simply a calculation in $\mathrm{C} \ell(\mathrm{V})$. Let us prove first the identity (36). In the proof of Lemma 15 we showed that

$$
\delta(v, w)=8 \theta\left(v_{-}\right)\left(4 w_{\top}+w_{\perp}\right) \cdot \varphi \cdot f-8 \theta\left(w_{-}\right)\left(4 v_{\top}+v_{\perp}\right) \cdot \varphi \cdot f,
$$

where we recall that $\theta\left(v_{-}\right)=\eta\left(\nu, e_{+}\right)$. Therefore in $\mathrm{C} \ell(V)$,

$$
\begin{aligned}
{[\delta(v, w), u]=} & {\left[\delta(v, w), u_{\top}+u_{\perp}+u_{-}+u_{+}\right] } \\
= & +8 \theta\left(v_{-}\right)\left[\left(4 w_{\top}+w_{\perp}\right) \cdot \varphi \cdot f, u_{\top}+u_{\perp}+u_{-}\right] \\
& -8 \theta\left(w_{-}\right)\left[\left(4 v_{\top}+v_{\perp}\right) \cdot \varphi \cdot f, u_{\top}+u_{\perp}+u_{-}\right]
\end{aligned}
$$

where we have used that $\delta(v, w)$ leaves $\boldsymbol{e}_{+}$invariant. Next we use the following results:

$$
\begin{array}{ll}
{\left[w_{\top} \cdot \varphi \cdot f, u_{\top}\right]=2 \eta\left(w_{\top}, u_{\top}\right) \varphi \cdot f,} & {\left[w_{\perp} \cdot \varphi \cdot f, u_{\top}\right]=0} \\
{\left[w_{\perp} \cdot \varphi \cdot f, u_{\perp}\right]=2 \eta\left(w_{\perp}, u_{\perp}\right) \varphi \cdot f,} & {\left[w_{\top} \cdot \varphi \cdot f, u_{-}\right]=-2 \theta\left(u_{-}\right) w_{\top} \cdot f^{2}} \\
{\left[w_{\top} \cdot \varphi \cdot f, u_{\perp}\right]=0,} & {\left[w_{\perp} \cdot \varphi \cdot f, u_{-}\right]=-2 \theta\left(u_{-}\right) w_{\perp} \cdot f^{2},}
\end{array}
$$

and arrive at

$$
\begin{aligned}
\frac{1}{8}[\delta(v, w), u]= & 8 \theta\left(v_{-}\right) \eta\left(w_{\top}, u_{\top}\right) \varphi \cdot f-8 \theta\left(w_{-}\right) \eta\left(v_{\top}, u_{\top}\right) \varphi \cdot f \\
& +2 \theta\left(v_{-}\right) \eta\left(w_{\perp}, u_{\perp}\right) \varphi \cdot f-2 \theta\left(w_{-}\right) \eta\left(v_{\perp}, u_{\perp}\right) \varphi \cdot f \\
& -8 \theta\left(u_{-}\right) \theta\left(v_{-}\right) w_{\top} \cdot f^{2}+8 \theta\left(u_{-}\right) \theta\left(w_{-}\right) v_{\top} \cdot f^{2} \\
& -2 \theta\left(u_{-}\right) \theta\left(v_{-}\right) w_{\perp} \cdot f^{2}+2 \theta\left(u_{-}\right) \theta\left(w_{-}\right) v_{\perp} \cdot f^{2}
\end{aligned}
$$

which when inserted in the Jacobi identity vanishes, due to the terms cancelling pairwise, thus proving the identity (36). The identity (35) is proved in a similar way, so we will be brief. We now find that the left-hand side of (35) is given by

$$
-8 \theta\left(v_{-}\right)\left(4 z_{\top}+z_{\perp}\right) \Gamma_{+}+8 \theta\left(z_{-}\right)\left(4 v_{\top}+v_{\perp}\right) \Gamma_{+},
$$

and this is precisely what we obtain for the right-hand side.

Finally, the last claim of the theorem follows from Theorem 9. 
In summary, we find three isomorphism classes of nontrivial filtered deformations of $\mathbb{Z}$-graded subalgebras of the Poincaré superalgebra $\mathfrak{g}$ which differ only in degree zero. They are characterised by a decomposable $\varphi \in \Lambda^{4} V$. Such a $\varphi$ defines a stabiliser $\mathfrak{h}_{\varphi} \subset \mathfrak{s o}(\mathrm{V})$ and also a filtered deformation of the $\mathbb{Z}$-graded subalgebra $\mathfrak{h}_{\varphi} \oplus \mathrm{S} \oplus \mathrm{V} \subset \mathfrak{g}$ given by

$$
\begin{array}{ll}
{[A, B]=A B-B A,} & {[s, s]=[s, s]_{0}+t \gamma^{\varphi}(s, s)} \\
{[A, s]=A s,} & {[v, s]=t \beta^{\varphi}(v, s)} \\
{[A, v]=A v,} & {[v, w]=t^{2} \delta(v, w),}
\end{array}
$$

for all $A, B \in \mathfrak{h}_{\varphi}, s \in S$ and $\nu, w \in V$, and where the maps $\beta^{\varphi}: V \otimes S \rightarrow S$ and $\gamma^{\varphi}: \odot{ }^{2} S \rightarrow \mathfrak{h}_{\varphi}$ are as in Eq. (18) and $\delta: \Lambda^{2} \vee \rightarrow \mathfrak{h}_{\varphi}$ is as in Eq. (34).

By Lemma 13, CSO (V)-related $\varphi$ 's give rise to isomorphic filtered deformations, so it is enough to choose a representative $\varphi$ from each orbit. A possible choice is the following:

1. $\varphi=e_{0} \wedge e_{1} \wedge e_{2} \wedge e_{3}$, where $\left(e_{\mu}\right)$ is an $\eta$-orthonormal basis for $V$. The stabiliser is $\mathfrak{h}_{\varphi} \cong \mathfrak{s o}(1,3) \oplus \mathfrak{s o}(7)$. The Lie brackets on $\mathfrak{h}_{\varphi} \oplus \mathrm{V}$ can be read from Lemma 15, and we find that they give rise to a Lie algebra isomorphic to $\mathfrak{s o}(2,3) \oplus \mathfrak{s o}(8)$. This is the Lie algebra of isometries of the Freund-Rubin backgrounds $\mathrm{AdS}_{4} \times \mathrm{S}^{7}$. The resulting Lie superalgebra on $\mathfrak{h}_{\varphi} \oplus \mathrm{S} \oplus \mathrm{V}$ is isomorphic to the Killing superalgebra of this family of backgrounds; namely, $\mathfrak{o s p}(8 \mid 4)$.

2. $\varphi=\boldsymbol{e}_{7} \wedge \boldsymbol{e}_{8} \wedge \boldsymbol{e}_{9} \wedge \boldsymbol{e}_{\natural}$, where again $\left(\boldsymbol{e}_{\mu}\right)$ is an $\eta$-orthonormal basis for $\vee$. The stabiliser is $\mathfrak{h}_{\varphi} \cong \mathfrak{s o}(4) \oplus \mathfrak{s o}(1,6)$. The Lie brackets on $\mathfrak{h}_{\varphi} \oplus \mathrm{V}$ are isomorphic to $\mathfrak{s o}(5) \oplus \mathfrak{s o}(2,6)$, which is the isometry Lie algebra of the Freund-Rubin backgrounds $\mathrm{S}^{4} \times \mathrm{AdS}_{7}$. The resulting filtered deformation is isomorphic to the Lie superalgebra $\mathfrak{o s p}(2,6 \mid 4)$, which is the Killing superalgebra of this family of backgrounds.

3. $\varphi=e_{+} \wedge e_{1} \wedge e_{2} \wedge e_{3}$, relative to an $\eta$-Witt basis $\left(e_{+}, e_{-}, e_{i}\right)$ for $\bigvee$. The stabiliser is $\mathfrak{h}_{\varphi}=(\mathfrak{s o}(3) \oplus \mathfrak{s o}(6)) \ltimes \mathbb{R}^{9}$ and the Lie brackets on $\mathfrak{h}_{\varphi} \oplus \vee$ give it the structure of a Lie algebra isomorphic to the isometry Lie algebra of the Cahen-Wallach spacetime underlying the Kowalski-Glikman pp-wave. The resulting Lie superalgebra is isomorphic to the Killing superalgebra of the Kowalski-Glikman wave, which is itself a contraction (in the sense of Inönü-Wigner) of both of the Freund-Rubin Killing superalgebras.

In summary, we recover the classification of maximally supersymmetric vacua of 11-dimensional supergravity via their Killing superalgebras.

\section{Discussion and Conclusion}

In this paper we have determined the (isomorphism classes of) Lie superalgebras which are filtered deformations of $\mathbb{Z}$-graded subalgebras $\mathfrak{h}=\mathrm{V} \oplus \mathrm{S} \oplus \mathfrak{h}_{0}$, with $\mathfrak{h}_{0} \subset \mathfrak{s o}(\mathrm{V})$, of the 11-dimensional Poincaré superalgebra. We have found that aside from the Poincaré superalgebra itself $\left(\mathfrak{h}_{0}=\mathfrak{s o}(\mathrm{V})\right)$ and its $\mathbb{Z}$-graded subalgebras, there are three other Lie superalgebras corresponding to the symmetry superalgebras of the non-flat maximally supersymmetric backgrounds of 11-dimensional supergravity: the two (families of) Freund-Rubin backgrounds and their common Penrose limit.

In so doing we have recovered by cohomological means the connection $\mathrm{D}$ on the spinor bundle which is defined by the supersymmetry variation of the gravitino. We could say that we have, in a very real sense, rediscovered 11-dimensional supergravity from the Spencer cohomology of the Poincaré superalgebra. 
More remarkable still is perhaps the fact that the classification of nontrivial filtered deformations of subalgebras of the Poincare superalgebra precisely agrees with the classification of Killing superalgebras of non-flat maximally supersymmetric backgrounds of 11-dimensional supergravity. To be clear, what is remarkable is not that we recover these Killing superalgebras - after all, it can be shown in full generality that the symmetry superalgebra of a supersymmetric background is a filtered deformation of some subalgebra of the Poincaré superalgebra-but that we find no other filtered deformations. We interpret this as encouraging evidence as to the usefulness of both the notions of super Poincaré structures and of symmetry superalgebras as organisational tools in the classification problem of supersymmetric supergravity backgrounds.

An interesting question is whether every filtered deformation of a subalgebra of the Poincare superalgebra is geometrically realised as the Killing superalgebra of a supersymmetric background. First of all, as shown by the (undeformed) subalgebras of the Poincaré superalgebra, these are only contained in the maximal such superalgebra (namely, the Poincare superalgebra itself). This is not surprising since it is only the supertranslation ideal which is actually generated by the Killing spinors. More worrying, though, are examples of filtered deformations which are not yet known to be realised geometrically (such as the deformation of the M2-brane Killing superalgebra found in [41], which suggests very strongly the existence of a half-BPS black anti-de Sitter membrane, whose construction continues to elude us), or those such as the putative $\mathrm{N}=28$ pp-wave conjectured in [42] and which was shown in [5] not to exist.

Before concluding, we would also like to mention an interesting relation with the off-shell pure spinor superfield formulation of 11-dimensional supergravity (see, e.g., the review [43] and references therein). The starting point of the pure spinor approach is the observation that the bosonic equations of motion of 11-dimensional supergravity reside in the direct sum of irreducible $\mathfrak{s o}(\mathrm{V})$-modules with Dynkin labels [11000] and, respectively, [10002], cf. [43, Eq. (4.14)]. Now pure spinors are the Dirac spinors $s \in$ $\mathrm{S} \otimes \mathbb{C}$ with vanishing Dirac current. For them, the associated supercharge $\mathrm{Q}$ satisfies $\mathrm{Q}^{2}=0$ and one can see that the cohomology of $\mathrm{Q}$ encodes the (linearised) equations of motion.

In our approach one can check that the Spencer cohomology group $\mathrm{H}^{0,2}(\mathfrak{m}, \mathfrak{g})$ of the Poincaré superalgebra $\mathfrak{g}$ is isomorphic to [11000] $\oplus$ [10002], i.e., it encodes the equations of motion. This fact suggests the possibility of modifying the definition of a super Poincaré structure $(M, \mathcal{D})$ as a Tanaka structure whose "symbol space" $\mathfrak{m}(x)$ has been deformed along directions in $\mathrm{H}^{0,2}(\mathfrak{m}, \mathfrak{g})$. It might be interesting to investigate these more general Tanaka structures and understand differences and similarities with the pure spinor approach, also in view of possible applications to the construction of off-shell formulations of supergravity theories.

Acknowledgements. The research of JMF is supported in part by two Grants from the UK Science and Technology Facilities Council (ST/J000329/1 and ST/L000458/1), and that of AS is fully supported by a Marie-Curie research fellowship of the "Istituto Nazionale di Alta Matematica" (Italy). We are grateful to our respective funding agencies for their support. During the final stretch of writing, JMF was a guest of the Grupo de Investigación "Geometría diferencial y convexa" of the Universidad de Murcia, and he wishes to thank Ángel Ferrández Izquierdo for the invitation, the hospitality and for providing such a pleasant working atmosphere.

Open Access This article is distributed under the terms of the Creative Commons Attribution 4.0 International License (http://creativecommons.org/licenses/by/4.0/), which permits unrestricted use, distribution, and reproduction in any medium, provided you give appropriate credit to the original author(s) and the source, provide a link to the Creative Commons license, and indicate if changes were made. 


\section{Appendix A: Clifford Conventions}

The proofs of a couple of results are easier if we work relative to a basis for the Clifford algebra. In this appendix we set out the conventions which will be employed in this paper, especially to prove Proposition 7 and Lemma 11.

We start with some properties of the Clifford algebra associated to an 11-dimensional Lorentzian vector space $(\mathrm{V}, \eta)$ with "mostly minus" signature. The Clifford algebra $\mathrm{C} \ell(\mathrm{V})$, with relations

$$
v^{2}=-\eta(v, v) \mathbb{1} \quad \forall v \in \mathrm{V},
$$

is isomorphic as a real associative algebra to two copies of the algebra of real $32 \times 32$ matrices. It follows from this isomorphism that $\mathrm{C} \ell(\mathrm{V})$ has two inequivalent irreducible Clifford modules, which are real and of dimension 32.

The Clifford algebra $\mathrm{C} \ell(\mathrm{V})$ is filtered (and $\mathbb{Z}_{2}$-graded) and the associated graded algebra is the exterior algebra $\Lambda^{\bullet} \mathrm{V}$. An explicit vector space isomorphism $\Lambda^{\bullet} \mathrm{V} \cong \mathrm{C} \ell(\mathrm{V})$ can be described as follows.

Let $\left(\mathbf{e}_{\mu}\right)$, for $\mu=0,1, \ldots, 9, \natural$, be an $\eta$-orthonormal basis; that is,

$$
\eta\left(e_{\mu}, e_{v}\right)=\eta_{\mu \nu}=\left(\begin{array}{cc}
1 & 0 \\
0 & -I_{10}
\end{array}\right) .
$$

The Clifford algebra $\mathrm{C} \ell(\mathrm{V})$ is generated by the image of $\mathrm{V}$ under the map $\mathrm{V} \rightarrow \mathrm{C} \ell(\mathrm{V})$ which sends $\boldsymbol{e}_{\mu}$ to $\Gamma_{\mu}$, with

$$
\Gamma_{\mu} \Gamma_{\nu}+\Gamma_{\nu} \Gamma_{\mu}=-2 \eta_{\mu \nu} \mathbb{1}
$$

Notice that due to our choice of a mostly minus $\eta, \Gamma_{0}^{2}=-\mathbb{1}$. We use the notation $\Gamma_{\mu_{1} \ldots \mu_{p}}$ for the totally antisymmetric product

$$
\Gamma_{\mu_{1} \ldots \mu_{p}}=\Gamma_{\left[\mu_{1}\right.} \Gamma_{\mu_{2}} \ldots \Gamma_{\left.\mu_{p}\right]}:=\frac{1}{p !} \sum_{\sigma \in S_{p}}(-1)^{\sigma} \Gamma_{\mu_{\sigma(1)}} \ldots \Gamma_{\mu_{\sigma(p)}},
$$

with $S_{p}$ the symmetric group in $\{1, \ldots, p\}$ and $(-1)^{\sigma}$ the sign of the permutation $\sigma \in S_{p}$.

The explicit isomorphism $\Lambda^{\bullet} \mathrm{V} \rightarrow \mathrm{C} \ell(\mathrm{V})$ is built out of the maps $\wedge^{\mathrm{p}} \mathrm{V} \rightarrow \mathrm{C} \ell(\mathrm{V})$ given by sending

$$
\mathbf{e}_{\mu_{1}} \wedge \cdots \wedge e_{\mu_{p}} \mapsto \Gamma_{\mu_{1} \ldots \mu_{p}}
$$

and extending linearly. Thus an $\eta$-orthonormal basis for $\mathrm{V}$ induces a basis for $\mathrm{C} \ell(\mathrm{V})$ given by the $\Gamma_{\mu_{1} \ldots \mu_{p}}$ for $p=0,1, \ldots, 11$. The volume element $\Gamma_{11}=\Gamma_{0} \Gamma_{1} \ldots \Gamma_{\natural}$ is central in $\mathrm{C} \ell(\mathrm{V})$ and satisfies $\Gamma_{11} \Gamma_{11}=\mathbb{1}$. The two non-isomorphic irreducible Clifford modules $S_{ \pm}$of $C \ell(V)$ are distinguished by the action of $\Gamma_{11}$, where $\Gamma_{11}$ acts like $\pm \mathbb{1}$ on $S_{ \pm}$. We will work with $S=S_{-}$in this paper.

Endomorphisms of $\mathrm{S}$ can be described in terms of elements of $\mathrm{C} \ell(\mathrm{V})$. A basis for the endomorphisms of $S$ by the image in $\operatorname{End}(S)$ of $\Gamma_{\mu_{1} \ldots \mu_{p}}$ for $p=0,1, \ldots, 5$. We will often tacitly use this isomorphism

$$
\operatorname{End}(S) \cong \bigoplus_{p=0}^{5} \Lambda^{p} V
$$

in the paper and let $p$-forms with $p=0, \ldots, 5$ act on $S$. 
There is an action of $\mathfrak{s o}(\mathrm{V})$ on $\mathrm{S}$ via the embedding of $\mathfrak{s o}(\mathrm{V})$ in $\mathrm{C} \ell(\mathrm{V})$. This is described as follows. If $\mathrm{L}_{\mu \nu}=-\mathrm{L}_{\nu \mu} \in \mathfrak{s o}(\mathrm{V})$ is defined by

$$
\mathrm{L}_{\mu \nu}\left(\boldsymbol{e}_{\rho}\right)=\eta_{\rho \nu} \mathbf{e}_{\mu}-\eta_{\rho \mu} \boldsymbol{e}_{\nu}
$$

then it is embedded in $\mathrm{C} \ell(\mathrm{V})$ as

$$
\mathrm{L}_{\mu \nu} \mapsto-\frac{1}{2} \Gamma_{\mu \nu}=-\frac{1}{4}\left[\Gamma_{\mu}, \Gamma_{v}\right] .
$$

Indeed, we have the following commutator in $\mathrm{C} \ell(\mathrm{V})$ :

$$
\left[-\frac{1}{2} \Gamma_{\mu \nu}, \Gamma_{\rho}\right]=\eta_{\rho \nu} \Gamma_{\mu}-\eta_{\rho \mu} \Gamma_{\nu}
$$

On $S$ we have an $\mathfrak{s o}(\mathrm{V})$-equivariant symplectic structure $\langle-,-\rangle$. Relative to a Majorana basis for $S$ where the $\Gamma_{\mu}$ are represented by real matrices, we can choose the symplectic structure defined by the matrix representing $\Gamma_{0}$. If $s_{1}, s_{2} \in S$, it is often convenient to write $\left\langle s_{1}, s_{2}\right\rangle$ as $\overline{s_{1}} s_{2}$. We have that

$$
\overline{s_{1}} \Gamma_{\mu_{1} \ldots \mu_{\mathrm{p}}} s_{2}=\varepsilon_{\mathrm{p}} \overline{\mathrm{s}_{2}} \Gamma_{\mu_{1} \ldots \mu_{\mathrm{p}}} \mathrm{s}_{1},
$$

where $\varepsilon_{p}=+1$ for $p=1,2,5$ and $\varepsilon_{p}=-1$ for $p=0,3,4$, which reflects the isomorphisms of $\mathfrak{s o}(\mathrm{V})$-modules

$$
\Lambda^{2} S \cong \Lambda^{0} V \oplus \Lambda^{3} V \oplus \Lambda^{4} V \text { and } \odot^{2} S \cong V \oplus \Lambda^{2} V \oplus \Lambda^{5} V
$$

Three easy consequences of this fact are the following:

1. For $v \in \mathrm{V}$,

$$
\langle s, v \cdot s\rangle=0 \quad \forall s \in S \Longrightarrow v=0
$$

2. For $\zeta \in \Lambda^{2} \vee$

$$
\langle s, \zeta \cdot s\rangle=0 \quad \forall s \in S \Longrightarrow \zeta=0
$$

3. and for $\theta \in \Lambda^{5} \mathrm{~V}$,

$$
\langle s, \theta \cdot s\rangle=0 \quad \forall s \in S \Longrightarrow \theta=0 .
$$

Another consequence of this fact is the following isomorphism of $\mathfrak{s o}(\mathrm{V})$-modules

$$
\mathrm{S} \otimes \mathrm{S} \cong \bigoplus_{\mathrm{p}=0}^{5} \wedge^{\mathrm{p}} \mathrm{V},
$$

where the $\mathfrak{s o}(\mathrm{V})$-submodule of $\mathrm{S} \otimes \mathrm{S}$ isomorphic to $\Lambda^{\mathrm{q}} \mathrm{V}$ is given by

$$
\Lambda^{\mathrm{q}} \mathrm{V} \cong\left\{\sum s_{i} \otimes s_{i}^{\prime} \mid \sum \overline{s_{i}} \Gamma_{\mu_{1} \ldots \mu_{p}} s_{i}^{\prime}=0 \text { for all } p \neq q, 0 \leqslant p \leqslant 5\right\}
$$

for all $\mathrm{q}=0, \ldots, 5$ and that $\bar{s} \Gamma_{\mu_{1} \ldots \mu_{\mathrm{p}}} \mathrm{s}=0$ except when $\mathrm{p}=1,2,5$.

On occasion we will also need to use an $\eta$-Witt basis $\left(\boldsymbol{e}_{+}, \boldsymbol{e}_{-}, \boldsymbol{e}_{i}\right)$, with $i=1, \ldots, 9$, for $\mathrm{V}$, where $\eta\left(\boldsymbol{e}_{+}, \boldsymbol{e}_{-}\right)=1$ and $\eta\left(\boldsymbol{e}_{i}, \boldsymbol{e}_{j}\right)=-\delta_{i j}$. Given an $\eta$-orthonormal basis, we may obtain an $\eta$-Witt basis by $\mathbf{e}_{ \pm}=\frac{1}{\sqrt{2}}\left(\mathbf{e}_{0} \pm \mathbf{e}_{\natural}\right)$ and $\boldsymbol{e}_{1}, \ldots, \boldsymbol{e}_{9}$ coinciding. The image in $\mathrm{C} \ell(\mathrm{V})$ of $\boldsymbol{e}_{ \pm}$will be denoted $\Gamma_{ \pm}$and obey $\left(\Gamma_{ \pm}\right)^{2}=0$. 
Finally, we record a number of useful identities to perform calculations in the Clifford algebra.

If $\nu \in V$ and $\theta \in \Lambda^{p} V$ their Clifford product in $C \ell(V)$ satisfy

$$
\begin{aligned}
& v \cdot \theta=v \wedge \theta-\iota_{v} \theta \\
& \theta \cdot v=(-1)^{p}\left(v \wedge \theta+\iota_{v} \theta\right) .
\end{aligned}
$$

The Fierz identity expresses the rank-one endomorphism $s_{1} \overline{s_{2}}$ of $S$ defined by $\left(s_{1} \overline{s_{2}}\right)(s)=$ $\left\langle s_{2}, s\right\rangle s_{1}$ in terms of the standard basis of $\operatorname{End}(S)$. We shall only need the special case where $s_{1}=s_{2}$. The identity reads

$$
s \bar{s}=-\frac{1}{32}\left(\bar{s} \Gamma^{\mu} s \Gamma_{\mu}+\frac{1}{2} \bar{s} \Gamma^{\mu \nu} s \Gamma_{\mu \nu}+\frac{1}{5 !} \bar{s} \Gamma^{\mu_{1} \ldots \mu_{5}} s \Gamma_{\mu_{1} \ldots \mu_{5}}\right) .
$$

The following identities come in handy when using the Fierz identity:

$$
\begin{aligned}
\Gamma_{\mu} \Gamma_{v_{1} \ldots \nu_{p}} \Gamma^{\mu}= & (-1)^{p+1}(11-2 p) \Gamma_{v_{1} \ldots v_{p}} \\
\Gamma_{\mu_{1} \mu_{2}} \Gamma_{v_{1} \ldots v_{p}} \Gamma^{\mu_{1} \mu_{2}}= & \left(11-(11-2 p)^{2}\right) \Gamma_{v_{1} \ldots v_{p}} \\
\Gamma_{\mu_{1} \ldots \mu_{5}} \Gamma_{v_{1} \ldots v_{p}} \Gamma^{\mu_{1} \ldots \mu_{5}}= & (-1)^{p+1}\left((11-2 p)^{4}-90(11-2 p)^{2}+1289\right) \\
& (11-2 p) \Gamma_{v_{1} \ldots v_{p}},
\end{aligned}
$$

where $\Gamma^{\mu}$ is defined by $\Gamma_{v}=\eta_{\nu \mu} \Gamma^{\mu}$. It follows from these identities that

$$
\frac{1}{2} \bar{s} \Gamma_{\mu \nu} s \Gamma^{\mu \nu} s=5 \bar{s} \Gamma_{\mu} s \Gamma^{\mu} s \text { and } \frac{1}{5 !} \bar{s} \Gamma_{\mu_{1} \ldots \mu_{5}} s \Gamma^{\mu_{1} \ldots \mu_{5}} s=-6 \bar{s} \Gamma_{\mu} s \Gamma^{\mu} s,
$$

consistent with the fact that the endomorphism $s \bar{s}$ annihilates $s$ due to the symplectic nature of the spinor inner product.

\section{Appendix B: Some Representations of $\mathfrak{s o}(\mathrm{V})$}

The Lie algebra $\mathfrak{s o}(\mathrm{V})$ is a real form of the complex simple Lie algebra of type $\mathrm{B}_{5}$. We will therefore use the Dynkin label $\left[n_{1} \ldots n_{5}\right], n_{i} \in \mathbb{N}$, to refer to the (real) irreducible module with highest weight $\sum_{i} n_{i} \lambda_{i}$, where $\lambda_{i}$ are a choice of fundamental weights. The following dictionary is helpful. The module $V$ has Dynkin label [10000], whereas the adjoint module $\mathfrak{s o}(\mathrm{V}) \cong \Lambda^{2} \mathrm{~V}$ has label [01000] and the spinor module $S$ has label [00001]. Other representations which will play a rôle are shown in Table 5. The representations with a 0 subscript are the kernels of Clifford multiplication inside $V \otimes S$ or $V \otimes \Lambda^{p} V$ with $p \geqslant 1$. In other words, they are the irreducible $\mathfrak{s o}(V)$-modules defined by the short exact sequences:

$$
\begin{aligned}
& 0 \rightarrow(\mathrm{V} \otimes \mathrm{S})_{0} \rightarrow \mathrm{V} \otimes \mathrm{S} \stackrel{\mathrm{cl}}{\rightarrow} \mathrm{S} \rightarrow 0 \\
& 0 \rightarrow\left(\mathrm{V} \otimes \Lambda^{\mathrm{p}} \mathrm{V}\right)_{0} \rightarrow \mathrm{V} \otimes \Lambda^{\mathrm{p}} \mathrm{V} \stackrel{\mathrm{cl}}{\rightarrow} \Lambda^{\mathrm{p}-1} \mathrm{~V} \oplus \Lambda^{\mathrm{p}+1} \mathrm{~V} \rightarrow 0,
\end{aligned}
$$

where $\Lambda^{1} V=V$ and $\Lambda^{0} V=\mathbb{R}$. Notice that for $p=1$, there is an isomorphism of modules $\left(\mathrm{V} \otimes \Lambda^{1} \mathrm{~V}\right)_{0} \cong \odot_{0}^{2} \mathrm{~V}$, the $\eta$-traceless symmetric square of $\mathrm{V}$.

Open Access This article is distributed under the terms of the Creative Commons Attribution 4.0 International License (http://creativecommons.org/licenses/by/4.0/), which permits unrestricted use, distribution, and reproduction in any medium, provided you give appropriate credit to the original author(s) and the source, provide a link to the Creative Commons license, and indicate if changes were made. 
Table 5. Some irreducible modules of $\mathfrak{s o}(\mathrm{V})$

\begin{tabular}{llr}
\hline Label & Alias & Dim \\
\hline$[00000]$ & $\mathbb{R}$ & 1 \\
{$[10000]$} & $\vee$ & 11 \\
{$[00001]$} & $\mathrm{S}$ & 32 \\
{$[01000]$} & $\wedge^{2} \mathrm{~V}$ & 55 \\
{$[00100]$} & $\wedge^{3} \mathrm{~V}$ & 165 \\
{$[00010]$} & $\wedge^{4} \mathrm{~V}$ & 330 \\
{$[00002]$} & $\wedge^{5} \mathrm{~V}$ & 462 \\
{$[10001]$} & $(\vee \otimes S)_{0}$ & 320 \\
{$[20000]$} & $\odot_{0}^{2} \vee$ & 65 \\
{$[11000]$} & $\left(\vee \otimes \wedge^{2} \mathrm{~V}\right)_{0}$ & 429 \\
{$[10100]$} & $\left(\vee \otimes \wedge^{3} \mathrm{~V}\right)_{0}$ & 1430 \\
{$[10010]$} & $\left(\vee \otimes \wedge^{4} \mathrm{~V}\right)_{0}$ & 3003 \\
{$[10002]$} & $\left(\vee \otimes \wedge^{5} \mathrm{~V}\right)_{0}$ & 4290 \\
\hline
\end{tabular}

\section{References}

1. Duff, M., Nilsson, B., Pope, C.: Kaluza-Klein supergravity. Phys. Rep. 130, 1-142 (1986)

2. Figueroa-O'Farrill, J.M., Papadopoulos, G.: Maximally supersymmetric solutions of ten- and elevendimensional supergravity. J. High Energy Phys. 03, 048 (2003). arXiv:hep-th/0211089

3. Gran, U., Gutowski, J., Papadopoulos, G., Roest, D.: N = 31, D = 11. J. High Energy Phys. 02, 043 (2007). arXiv:hep-th/0610331

4. Figueroa-O'Farrill, J.M., Gadhia, S.: M-theory preons cannot arise by quotients. J. High Energy Phys. 06, 043 (2007). arXiv:hep-th/0702055

5. Gran, U., Gutowski, J., Papadopoulos, G.: M-theory backgrounds with 30 Killing spinors are maximally supersymmetric. JHEP 1003, 112 (2010). arXiv:1001.1103 [hep-th]

6. Gauntlett, J.P., Pakis, S.: The geometry of D = 11 Killing spinors. J. High Energy Phys. 04, 039 (2003). arXiv:hep-th/0212008

7. Gauntlett, J.P., Gutowski, J., Pakis, S.: The geometry of D=11 null Killing spinors. J. High Energy Phys. 12, 049 (2003). arXiv:hep-th/0311112

8. Michelson, J.: “A pp wave with twenty six supercharges”. Class. Quant. Grav. 19, 5935-5949 (2002). arXiv:hep-th/0206204

9. Kruglikov, B., The, D.: The gap phenomenon in parabolic geometries. J. Reine Angew. Math. (2014) arXiv:1303.1307 [math.DG]

10. de Medeiros, P.: Submaximal conformal symmetry superalgebras for Lorentzian manifolds of low dimension. J. High Energy Phys. 02, 008 (2016). arXiv:1406.5166 [hep-th]

11. Figueroa-O'Farrill, J., Hustler, N.: The homogeneity theorem for supergravity backgrounds. JHEP 1210, 014 (2012). arXiv: 1208.0553 [hep-th]

12. Figueroa-O'Farrill, J.: Symmetric M-Theory Backgrounds. Cent. Eur. J. Phys. 11, 1-36 (2013). arXiv:1112.4967 [hep-th]

13. Hustler, N., Lischewski, A.: "(M-theory-)Killing spinors on symmetric spaces". J. Math. Phys. 56(8), 082303 (2015). arXiv:1503.05350 [hep-th]

14. Figueroa-O'Farrill, J., Ungureanu, M.: Homogeneous M2 duals. JHEP 01, 150 (2016). arXiv:1511.03637 [hep-th]

15. Lischewski, A.: Computation of generalized Killing spinors on reductive homogeneous spaces. arXiv:1409.2664 [math.DG]

16. Cheng, S.-J., Kac, V.G.: Generalized Spencer cohomology and filtered deformations of $\mathbb{Z}$-graded Lie superalgebras. Adv. Theor. Math. Phys. 2(5), 1141-1182 (1998)

17. Cheng, S.-J., Kac, V.: "Addendum: "Generalized Spencer cohomology and filtered deformations of $\mathbb{Z}$ graded Lie superalgebras. Adv. Theor. Math. Phys. 8(4), 697-709 (2004) [Adv. Theor. Math. Phys. 2(5), 1141-1182 (1998); mr1688484]

18. Nahm, W.: Supersymmetries and their representations. Nucl. Phys. B 135, 149-166 (1978)

19. Cremmer, E., Julia, B., Scherk, J.: Supergravity in eleven dimensions. Phys. Lett. 76, 409-412 (1978)

20. Freund, P., Rubin, M.: Dynamics of dimensional reduction. Phys. Lett. B 97, 233-235 (1980)

21. Pilch K., van Nieuwenhuizen P., Townsend P.K. Compactification of $d=11$ supergravity on $S^{4}$ (or $11=$ $7+4$, too). Nucl. Phys. B 242, 377 (1984) 
22. Kowalski-Glikman, J.: Vacuum states in supersymmetric Kaluza-Klein theory. Phys. Lett. 134, 194196 (1984)

23. Blau, M., Figueroa-O'Farrill, J.M., Hull, C.M., Papadopoulos, G.: Penrose limits and maximal supersymmetry. Class. Quant. Grav. 19, L87-L95 (2002). arXiv:hep-th/0201081

24. Figueroa-O'Farrill, J.M.: On the supersymmetries of Anti-de Sitter vacua. Class. Quant. Grav. 16, 20432055 (1999). arXiv:hep-th/9902066

25. Figueroa-O'Farrill, J.M., Papadopoulos, G.: Homogeneous fluxes, branes and a maximally supersymmetric solution of M-theory. J. High Energy Phys. 06, 036 (2001). arXiv:hep-th/0105308

26. Hatsuda, M., Kamimura, K., Sakaguchi, M.: Super-pp-wave algebra from super-AdS $\times S$ algebras in eleven-dimensions. Nucl. Phys. B 637, 168-176 (2002). arXiv:hep-th/0204002

27. Brink, L., Howe, P.S.: Eleven-dimensional supergravity on the mass-shell in superspace. Phys. Lett. B 91, 384 (1980)

28. Cremmer, E., Ferrara, S.: Formulation of eleven-dimensional supergravity in superspace. Phys. Lett. B 91, 61 (1980)

29. Lott, J.: Torsion constraints in supergeometry. Comm. Math. Phys. 133(3), 563-615 (1990)

30. Howe, P.S.: "Weyl superspace". Phys. Lett. B 415, 149-155 (1997). arXiv:hep-th/9707184

31. Guillemin, V.: The integrability problem for G-structures. Trans. Am. Math. Soc. 116, 544-560 (1965)

32. Santi, A., Spiro, A.: Super-Poincaré algebras, space-times and supergravities (I). Adv. Theor. Math. Phys. 16(5), 1411-1441 (2012)

33. Santi, A., Spiro, A.: "Super-Poincaré algebras, space-times, and supergravities. II". J. Math. Phys. 53(3), 032505 (2012)

34. Tanaka, N.: On differential systems, graded Lie algebras and pseudogroups. J. Math. Kyoto Univ. 10, 1$82(1970)$

35. Tanaka, N.: On the equivalence problems associated with simple graded Lie algebras. Hokkaido Math. J. 8(1), 23-84 (1979)

36. Altomani, A., Santi, A.: Tanaka structures modeled on extended Poincaré algebras. Indiana Univ. Math. J. 63(1), 91-117 (2014)

37. Altomani, A., Santi, A.: Classification of maximal transitive prolongations of super-Poincaré algebras. Adv. Math. 265, 60-96 (2014)

38. Kostant, B.: Lie algebra cohomology and the generalized Borel-Weil theorem. Ann. Math. 74, 329387 (1961)

39. Bryant, R.L.: Pseudo-Riemannian metrics with parallel spinor fields and vanishing Ricci tensor. In: Bourgignon, J.P., Branson, T., Hijazi, O. (eds.) Global analysis and harmonic analysis (MarseilleLuminy, 1999), vol. 4 of Sémin. Congr., pp. 53-94. Soc. Math. France, Paris (2000). arXiv:math/0004073 [math.DG]

40. Griffiths, P., Harris, J.: Principles of algebraic geometry. Pure and Applied Mathematics. WileyInterscience [John Wiley \& Sons], New York (1978)

41. Figueroa-O'Farrill, J.M.: Deformations of M-theory Killing superalgebras. Class. Quant. Grav. 24, 52575276 (2007). arXiv:0706.2600 [hep-th]

42. Fernando, S., Gunaydin, M., Hyun, S.: Oscillator construction of spectra of PP-wave superalgebras in eleven dimensions. Nucl. Phys. B 727, 421-460 (2005). arXiv:hep-th/0411281

43. Cederwall, M.: Pure spinor superfields-an overview. Springer Proc. Phys. 153, 61-93 (2014). arXiv:1307.1762 [hep-th]

Communicated by X. Yin 